\title{
TAFONOMÍA Y ANÁLISIS SECUENCIAL DEL PLIOCENO INFERIOR EN EL SECTOR NE DE LA CUENCA DE ALMERÍA-NÍJAR (SE DE ESPAÑA)
}

\author{
Julio AGUIRRE y Jesús YESARES-GARCÍA \\ Dpto. Estratigrafía y Paleontología, Facultad de Ciencias, Fuentenueva, s/n, \\ Universidad de Granada, 18071 Granada, España. \\ 'E-mail: jaguirre@goliat. urg.es
}

Aguirre, J. y Yesares-García, J. 2003. Tafonomía y análisis secuencial del Plioceno Inferior en el sector NE de la cuenca de Almería-Níjar (SE de España). [Taphonomy and sequence stratigraphy of the Lower Pliocene deposits of the NE sector of the Almería-Níjar basin (SE Spain).] Revista Española de Paleontología, 18(1), 61-82. ISSN 0213-6937.

\begin{abstract}
Taphonomic attributes of fossils of the lower Pliocene deposits of the NE edge of the Almería-Níjar basin (SE Spain) has been analysed following a quantitative method. This, together with both facies analysis and fossil assemblages, allows to establishing palaeoenvironmental settings in which these sediments were deposited. Two stratigraphic sections has been studied. In both sections, the Pliocene unit starts with massive silts and finegrained sands that change to medium-grained sands at the mid part. In the upper half, three characteristic beds of calcarenites-calcirudites with trough cross-bedding are intercalated in the massive sands. With respect to the fossil assemblages, the base of the sequence is dominated by organisms of deep, low-energy conditions: Korobkovia oblonga, Amusium cristatum and Neopycnodonte cochlear. These sediments are interpreted as outershelf deposits formed below the storm wave-base. In the middle part of the sequence, fossil content is higher and fossil associations are more diverse. They are characterised by the pectinids Flabellipecten bosniaskii, Chlamys spp., Pecten jacobaeus, bryozoans (rigid erect colonies, free-living lunulitiform colonies and sphaeroidal colonies), the worm-tube serpulid Ditrupa, barnacles, and echinoids (cydarids and Clypeaster). Carbonate beds are dominated by typical shallow nearshore organisms, such as Ostrea lamellosa, pectinids with high strength ribbing and curvature (Gigantopecten and Flabellipecten), moulds of Isognomon (Hippochaeta) maxilatus, bryozoans and barnacles. These beds are attributed to shallow inner-shelf sediments deposited above the storm wave-base. In relation with the taphonomic attributes, the silts and sands of the outer-shelf settings are characterised by a low percentage of bioclasts per rock volume, a great dispersion of the size-frequency distribution (sorting), a low degree of abrasion (predomination of fragments with sharp edges), and a bimodal biofabric (abundance of concordantly and perpendicularly oriented shells). Fossil concentrations within burrows occuring in the sands are represented by a high concentration of fossils, most of them with sharp edges and with relatively high proportion of interactions, and abundance of concave-up oriented shells. Finally, inner-shelf facies are distinguished by a high degree of fragmentation, abrasion and disarticulation. In addition, they show a high dispersion of shell sizes and domination of concordantly oriented fossils.

Palaeoenvironmental interpretation, together with the geometry of the sediments, allows to inferring a sequence stratigraphic model. Thus, the outer-shelf deposits of the lower half part of the sequence represent the transgressive systems tract. On the other hand, the carbonate beds of the upper part represent the progradation of the inner platform during the depositon of the highstand systems tract. The characteristic alternation of sands and calcarenites-calcirudites is attributed to a higher frequency cyclicity.
\end{abstract}

Keywords: Taphonomy, sequence analysis, Early Pliocene, Almería-Níjar basin.

\section{RESUMEN}

Las propiedades tafonómicas de los fósiles contenidos en los materiales del Plioceno Inferior del sector NE de la cuenca de Almería-Níjar han sido analizadas mediante un estudio cuantitativo. Esto, junto con el análisis de facies y de las asociaciones de organismos, permite inferir las condiciones paleoambientales en las que se formaron dichos sedimentos. Se han estudiado dos secciones estratigráficas que constan de un tramo inferior que comienza con limos y arenas finas que cambian a arenas medias hacia la mitad de la secuencia. Por encima, tres 
paquetes de calcarenitas-calcirruditas con laminaciones y estratificaciones cruzadas se intercalan entre arenas masivas. En cuanto a las asociaciones de fósiles, en la base dominan organismos típicos de ambientes profundos y de baja energía, como Korobkovia oblonga, Amusium cristatum y Neopycnodonte cochlear. Estos materiales se interpretan como depósitos de plataforma externa formados por debajo del oleaje de tormentas. Hacia la mitad de la secuencia, aumenta el contenido en fósiles y las asociaciones se hacen más diversas y están dominadas por pectínidos como Flabellipecten bosniaskii, Chlamys spp., Pecten jacobeus, briozoos (colonias con crecimiento erecto rígido, colonias lunulitiformes de vida libre y colonias esferoidales), el serpúlido Ditrupa, balánidos y equinodermos (cidáricos y Clypeaster). Los niveles carbonatados están caracterizados por organismos típicos de medios someros, como Ostrea lamellosa, pectínidos con una fuerte costulación y concavidad de la valva (Gigantopecten y Flabellipecten), moldes de Isognomon (Hippochaeta) maxilatus, briozoos con crecimiento erecto rígido, equinodermos y balánidos. Se corresponden con depósitos de plataforma interna por encima del nivel del oleaje de tormentas. En cuanto a las propiedades tafonómicas, los limos y arenas de plataforma externa están caracterizados por un bajo porcentaje de bioclastos por volumen de roca, una alta proporción de bioclastos con las aristas agudas, una gran dispersión de frecuencias de tamaños y una cierta bimodalidad en la orientación de los fósiles (concordante y perpendicular). Dentro de las arenas se diferencian concentraciones fosilíferas en rellenos de madrigueras, representadas por una alta concentración de bioclastos predominantemente con las aristas agudas, dominio de restos orientados con la concavidad hacia arriba y un porcentaje de interacciones relativamente alto. Finalmente, las facies de plataforma interna se distinguen por poseer un alto grado de fragmentación, desarticulación y abrasión. Asimismo, presentan una alta dispersión en los tamaños de los fragmentos y un dominio de conchas orientadas con un ángulo inferior a $30^{\circ}$ (concordantes).

La interpretación paleoambiental, junto con la geometría de los materiales, permiten proponer un modelo de evolución secuencial. Así, el tramo inferior, constituido por limos y arenas de plataforma externa, representa los depósitos del cortejo sedimentario transgresivo. Por su parte, el tramo superior, donde se intercalan los bancos carbonatados, se corresponde con los depósitos del cortejo sedimentario de alto nivel del mar. Internamente, la alternancia arenas-paquetes carbonatados refleja una ciclicidad de alta frecuencia.

Palabras clave: Tafonomía, análisis secuencial, Plioceno Inferior, cuenca de Almería-Níjar.

\section{INTRODUCCIÓN}

La tafonomía ha sufrido cambios substanciales en sus líneas de investigación desde su nacimiento como disciplina paleontológica. Así, en los últimos treinta años se ha pasado a considerar que, a pesar de que los cambios post-mortem son los primeros en provocar una huella tafonómica causante de una pérdida de información, el enterramiento y la preservación proporcionan una información adicional sobre la génesis de los materiales que las partículas sedimentarias no ofrecen (Behrensmeyer y Kidwell, 1985; Brett y Baird, 1986; Speyer y Brett, 1986, 1991; Wilson, 1988; Behrensmeyer et al., 2000). Este nuevo enfoque de la tafonomía es el que perdura en la actualidad, de forma que se ha pasado de tener una visión negativa (pérdida de información) a una visión positiva (ganancia de información) de los procesos de fosilización (Behrensmeyer y Kidwell, 1985).

Un paso importante en los últimos años en relación con la información que puede extraerse de los procesos de fosilización ha sido la introducción del concepto de tafofacies (Speyer y Brett, 1986, 1991). Este concepto está directamente relacionado con lo que se ha denominado tafonomía comparada, que trata del estudio de la preservación diferencial de los fósiles (Brett y Baird, 1986). Es decir, los restos fósiles se comportan de distinta forma ante los diferentes procesos bioestratinómicos y fosildiagenéticos tempranos dependiendo de las condiciones ambientales de partida, de la organización esquelética de los organismos y de su modo de vida (Speyer y Brett, 1986, 1991). Esto implica que el modo de preservación puede aportar una información muy valiosa sobre los mecanismos de depósito en los ambientes en los que fosilizaron los organismos.

Por otro lado, la superposición a lo largo del tiempo de las diferentes tafofacies permite establecer un modelo evolutivo de las condiciones ambientales (Speyer y Brett, 1988). Este hecho, junto con el estudio estratigráfico de las concentraciones de fósiles, convierten al análisis tafonómico y de tafofacies en una herramienta esencial en la estratigrafía secuencial (Kidwell, 1988, 1989, 1991a, 1991b, 1993; Speyer y Brett, 1988; Holland, 1995; Brett, 1995, 1998; Aguirre, 1996). En definitiva, el estudio de los estilos de preservación de los fósiles, junto con el análisis de las asociaciones de fósiles y de las facies, permite establecer patrones de evolución paleoambiental y secuencial muy precisos.

Siguiendo en esa línea de renovado interés en tafonomía para la reconstrucción de los medios antiguos, en este trabajo presentamos un estudio tafonómico de las asociaciones de fósiles del Plioceno Inferior de la zona de El Argamasón, en el extremo NE de la cuenca de Almería-Níjar (SE de España). Las interpretaciones paleoambientales están basadas en el estudio cuantitativo de las propiedades tafonómicas de los fósiles siguiendo la metodología propuesta recientemente para materiales neógenos (Yesares-García y Aguirre, 2002). Esto permite inferir qué condiciones ambientales son las que ejercen un mayor control en el tipo de preservación de los fósiles (tipo de substrato, energía del agua, turbulencia, 
profundidad, etc.). Estas interpretaciones, apoyadas con criterios de la arquitectura estratigráfica de los materiales estudiados, sirven de base para proponer un modelo de evolución secuencial de los materiales estudiados.

\section{LOCALIZACIÓN GEOGRÁFICA Y CONTEXTO GEOLÓGICO}

El afloramiento estudiado se localiza en el extremo NE de la cuenca de Almería-Níjar. Se sitúa aproximadamente $1 \mathrm{~km}$ al NE de El Argamasón (Fig. 1), una pedanía perteneciente al término municipal de Carboneras. Dicho afloramiento comprende los relieves denominados Los Ranchos y La Loma de Ciscar, que forman una alineación topográfica $\mathrm{N}-\mathrm{S}$ que se extiende a lo largo de $1 \mathrm{~km}$. Se han realizado dos secciones estratigráficas localizadas en los extremos $\mathrm{S}$ y $\mathrm{N}$ de Los Ranchos, Los Ranchos I y Los Ranchos II respectivamente (Figs. 1 y 2).

La cuenca de Almería-Níjar está limitada por Sierra de Gádor al O, Sierra Alhamilla y Sierra Cabrera al N, y Sierra de Gata al E. Estos relieves constituyen el basamento de la cuenca, el cual está formado por rocas metamórficas y carbonatadas del Permo-Trías en el $\mathrm{O}$ y $\mathrm{N}$, y por rocas volcánicas del Mioceno en el E. Hacia el $\mathrm{S}$, la cuenca se abre al Mar Mediterráneo (Aguirre, 1998; Braga et al., 2001, 2003).

El relleno sedimentario de la cuenca está integrado por distintos conjuntos discordantes de materiales neógenos que comienza con margas de edad Mioceno medio (Serrano, 1979; Montenat et al., 1990) y finaliza con depósitos mixtos terrígeno-carbonatados del Plioceno Superior (Aguirre, 1998). Por encima de los materiales neógenos, se disponen discordantemente depósitos de playa y de abanicos aluviales atribuidos al Cuaternario (Goy y Zazo, 1982, 1986). Dentro de este conjunto neógeno, los depósitos pertenecientes al Plioceno se pueden dividir en dos unidades, denominadas Unidades I y II, separadas por una discordancia (Aguirre, 1998).

En la zona de El Argamasón (Fig. 1), el Plioceno está representado únicamente por la unidad inferior, Unidad I, la cual se dispone discordantemente sobre sedimentos del Messiniense y sobre el substrato volcánico. En la zona de estudio el contacto Mioceno/Plioceno está caracterizado por una superficie intensamente bioturbada, donde las galerías penetran en las margas miocenas hasta una profundidad máxima de $50 \mathrm{~cm}$ desde el contacto. Los sedimentos pliocenos correspondientes a la Unidad I se depositaron adaptándose a un paleorrelieve, produciéndose cambios de espesores importantes y transiciones de facies más o menos rápidas. Así mismo, al E-SE de la zona de estudio pasa el accidente de Carboneras (Fig. 1), un sistema de fallas de dirección N35E a N40E (Montenat et al., 1990; Boorsma, 1992). Durante el Plioceno se produjo el bloqueo del accidente de Carboneras y la formación de pliegues con ejes aproximadamente E-O y de fallas, cuya actividad se prolongó hasta el Pleistoceno (Montenat et al., 1990).

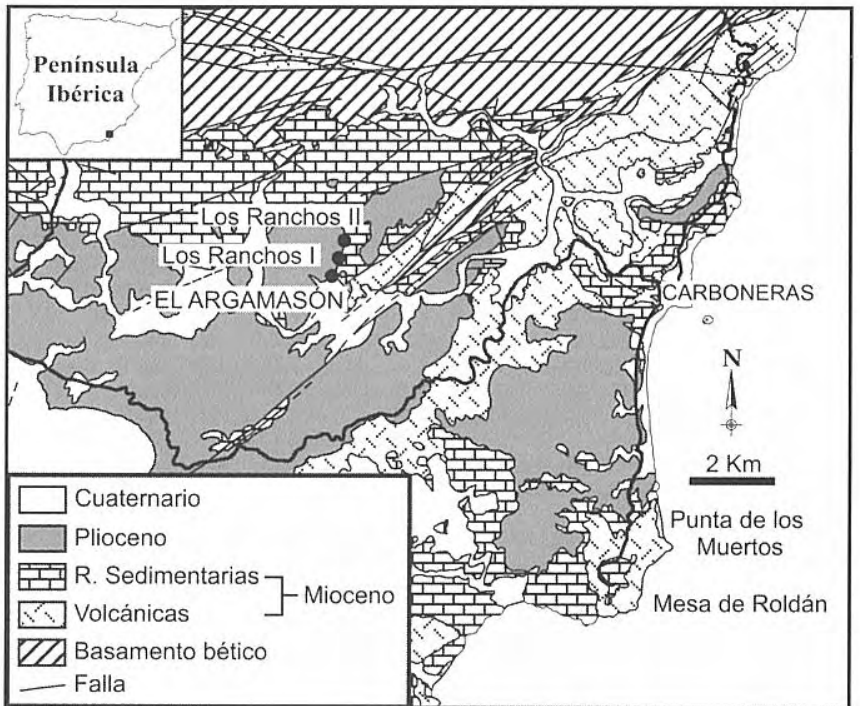

Figura 1. Mapa geológico de la zona de estudio y localización de las secciones estudiadas. Geological map of the study area indicating the location of the two studied sections.

\section{SECCIÓN DE LOS RANCHOS I}

\section{ESTRATIGRAFÍA Y ASOCIACIONES DE FÓSILES}

Esta sección se puede dividir en dos tramos, uno inferior constituido por un conjunto de sedimentos masivos y otro superior donde se intercalan diversos cuerpos carbonatados (Fig. 2). El tramo inferior comienza con limos y arenas muy finas de color amarillento, masivas y poco cementadas. En esta parte basal de la sección se encuentran cantos de rocas volcánicas dispersos. La bioturbación aparece fundamentalmente dispersa (ii2 a ii3 según los índices de icnofábricas de Droser y Bottjer, 1986, 1989), aunque también se observan niveles con una bioturbación más intensa (ii4) de trazas horizontales. En estos materiales los fósiles, aunque escasos, están dominados por los bivalvos Korobkovia oblonga y Chlamys spp., y por el gasterópodo Scalaria. Ocasionalmente se encuentra algún ejemplar de balánido.

Según se sube en este tramo inferior (hacia la mitad de la unidad) se produce un enriquecimiento en fósiles, al mismo tiempo que se acentúa el relieve debido a una mayor cementación del sedimento. Así mismo, se observa un aumento en el tamaño de grano, un cambio en el color a tonalidades más blanquecinas y un mayor contenido en micas. Además, los cantos volcánicos desaparecen hacia la mitad de la sección y comienzan a aparecer cantos de naturaleza metamórfica (mayoritariamente cantos de cuarcita). En cuanto a la fauna aparecen briozoos, bivalvos y equinodermos.

Antes de la intercalación del primer paquete carbonatado que da paso al inicio del segundo tramo, se observa un nivel de algas coralináceas formando rodolitos con un espesor de unos $10 \mathrm{~cm}$ (Fig. 2). Se trata de un nivel bien definido que puede seguirse en 
Los Ranchos I

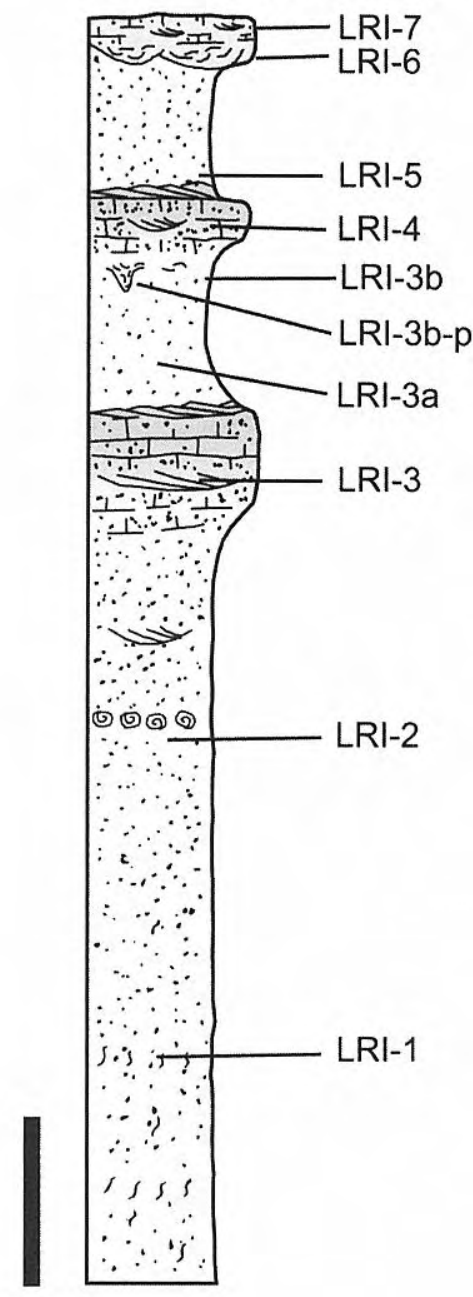

Los Ranchos II

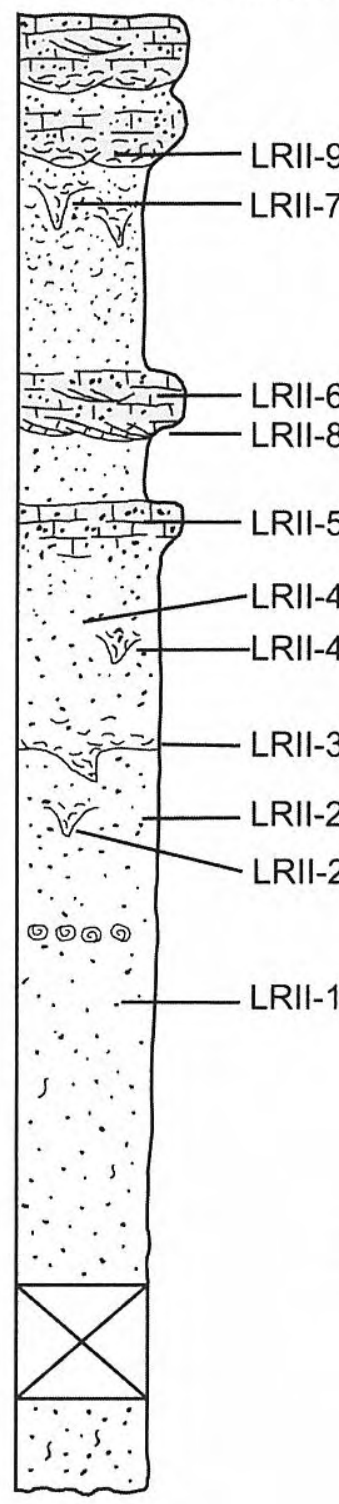

$\because \because \quad$ Limos y arenas

$\because \cdot{ }_{1}$ Calcarenitas/calcirruditas

$\leadsto$ Laminación cruzada en artesa

s s Bioturbación

Rellenos de madrigueras

๑๑ه Algas coralináceas (rodolitos)

Figura 2. Columnas estratigráficas de las secciones Los Ranchos I y Los Ranchos II. Las flechas indican la posición de las estaciones de muestreo. Escala vertical $=10 \mathrm{~m}$.

Stratigrafic columns of the Los Ranchos I and Los Ranchos II sections. Arrows point out the position of the sampling sites. Vertical scale $=10 \mathrm{~m}$.

continuidad lateral a lo largo de aproximadamente $500 \mathrm{~m}$ entre las dos secciones estudiadas. Junto a las algas se encuentran también numerosos restos fósiles, dominados por Spondylus, Neopycnodonte, Ostrea, Pecten, Chlamys, Clypeaster, equinodermos regulares (cidáridos) y fragmentos de mamíferos marinos.

Inmediatamente por encima del nivel de rodolitos aparecen laminaciones cruzadas en artesa en las arenas. Estas se pueden seguir lateralmente hacia el $\mathrm{S}$ y un poco hacia el N, desapareciendo en este sentido. Por encima se pasa nuevamente a arenas masivas en las que se aprecia una estratificación horizontal muy burda. Aquí se pueden encontrar pequeñas concentraciones de bioclastos dispersas en las que se encuentra principalmente Neopycnodonte cochlear.

El primer tramo culmina con un paquete de calcarenitas, denominado Calcarenita I (Fig. 2), de espesor variable y con laminaciones cruzadas en artesa que marcan una dirección de paleocorriente hacia el N. El contacto inferior con las arenas es gradual. Lateralmente hacia el S, hacia la Loma de Ciscar, las calcarenitas pasan gradualmente a arenas muy bioclásticas $\mathrm{y}$, finalmente, a arenas con laminaciones cruzadas. Los fósiles más abundantes son algas coralinas, briozoos, pectínidos (entre los que se encuentran Pecten jacobaeus, Flabellipecten bosniasckii y Chlamys varia), balánidos y equinodermos.

Inmediatamente por encima de la Calcarenita I se encuentran unas arenas medias de color grisáceoamarillento, con laminaciones cruzadas en artesas, muy tendidas y de gran amplitud (Fig. 2). La fauna predominante en esta parte de la sección está representada, fundamentalmente, por fragmentos de equinodermos, Chlamys y otros pectínidos, como Flabellipecten bosniasckii y Flexopecten flexuosa, balánidos, ostreidos, briozoos y serpúlidos (Ditrupa).

Hacia el techo, estas arenas muestran un color amarillento verdoso y se hacen bastante micáceas y 
masivas. Predominan briozoos, equinodermos (cidáridos), algunos de los cuales llevan adosados balánidos, y Chlamys varia, Anomia y ostreidos (Neopycnodonte entre ellos). Dentro de este tramo arenoso aparecen acumulaciones en vaina ("pod") de bioclastos, una de las cuales ha sido muestreada para el análisis tafonómico.

De forma gradual se pasa al segundo paquete de calcarenitas (Calcarenita II, Fig. 2), también con laminaciones cruzadas en artesa que indican una migración de las estructuras hacia el N. Entre los bioclastos hay dispersos cantos metamórficos. La asociación faunística es similar a la encontrada en la Calcarenita I, aunque se aprecia un incremento en el contenido de Ostrea lamellosa. Destaca, además, la presencia de algunos individuos de Isognomon (Hippochaeta) maxilatus, los cuales aparecen como moldes.

Por encima aparecen de nuevo unas arenas de tamaño de grano medio, muy micáceas, que presentan un ordenamiento granocreciente a techo. Están muy poco cementadas en su extremo $\mathrm{S}$, mientras que lateralmente hacia el $\mathrm{N}$ y hacia el techo pasan a estar muy cementadas. Los organismos más abundantes en estas arenas son briozoos de crecimiento erecto, cuya abundancia es muy patente. Otros restos que se encuentran en la asociación son pectínidos, ostreidos de gran tamaño, Neopycnodonte con incrustaciones de serpúlidos, equinodermos (Clypeaster y cidáridos) y balánidos.

La sección acaba con un tercer paquete calcarenítico, denominado Calcarenita III (Fig. 2). Se trata de un cuerpo carbonatado con estratificación cruzada hacia el NO (N60E/15NO). Se caracteriza por presentar un contacto inferior neto y por la presencia de numerosas acumulaciones residuales ("lags") de bioclastos en la base de paleocanales y de los surcos de ripples. Son acumulaciones con gradación normal formadas por conchas de gran tamaño. Predominan los bivalvos, destacando especialmente pectínidos (Pecten jacobaeus, Flabellipecten bosniasckii, Chlamys) y ostreidos (Ostrea lamellosa y Neopycnodonte), junto con equinodermos y briozoos.

\section{RESULTADOS TAFONÓMICOS}

En la sección de Los Ranchos I se han establecido un total de 10 estaciones de muestreo. Los resultados numéricos de las propiedades tafonómicas se muestran en la Tabla 1. Considerando esta base de datos, y de acuerdo con la metodología propuesta por Yesares-García y Aguirre (2002), un análisis cluster en modo Q permite diferenciar cuatro grupos de muestras (Fig. 3).

En primer lugar, la agrupación A incluye exclusivamente a la estación de muestreo LRI-1, situada en las arenas de la base de la serie (Figs. 2, 3). Dicha agrupación difiere de todas las demás por tener los valores de abundancia más bajos $\left(0,004\right.$ bioclastos $\left./ \mathrm{cm}^{2}\right)$, el porcentaje de bioclastos completos más alto (12,5\%) y porque el $100 \%$ de los restos presentan las aristas agudas (Tabla 1). Finalmente, este grupo se caracteriza también

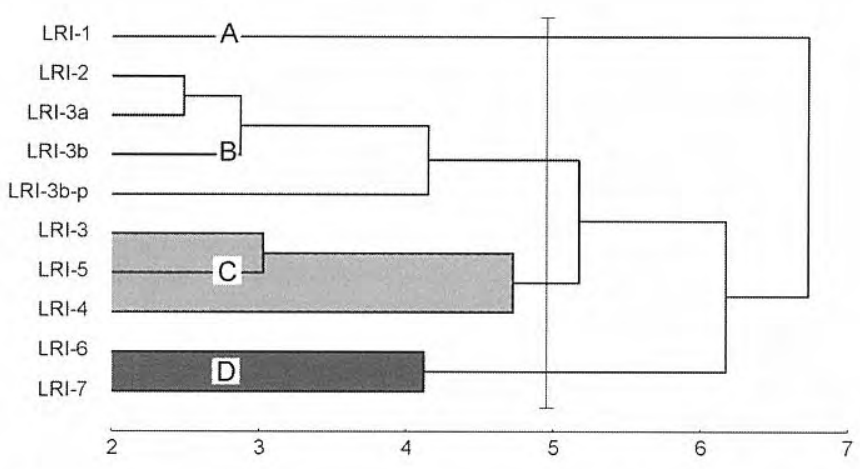

Figura 3. Análisis cluster de las propiedades tafonómicas medidas en la sección Los Ranchos I. Dendrograma obtenido a partir de un análisis cluster tipo Q usando el método del promedio simple y distancias euclídeas. La línea vertical marca la distancia más adecuada a la que se han realizado las agrupaciones según los métodos de Beale y Calinski.

Cluster analysis of the taphonomic attributes measured in Los Ranchos II section. Dendrogram obtained from a $Q$-mode cluster analysis, using unweigthted pair-group average method and Euclidean distances. The vertical line marks the most adequate distance to produce the groups according to the Beale and Calinski methods.

por presentar una gran dispersión en la distribución de frecuencias de tamaño (Fig. 4).

La agrupación B está formada por las estaciones LRI2, LRI-3a, LRI-3b y LRI-3b-p (Fig. 3). Todas estas estaciones tienen en común que están situadas en las arenas masivas (Fig. 2). Así mismo, incluye la única estación de muestreo situada en una acumulación de fósiles en vaina (LRI-3b-p). Todas las muestras incluidas en estas estaciones de muestreo, al igual que ocurría con la agrupación $\mathrm{A}$, se caracterizan por poseer valores muy bajos en el porcentaje de bioclastos por volumen de roca (Tabla 1). Con respecto a la orientación de los fósiles, estas estaciones muestran una distribución de ángulos bimodal, predominando las orientaciones concordantes y perpendiculares respecto al plano de estratificación. En consecuencia, muestran también los porcentajes de conchas en posición vertical más altos. Por otro lado, no hay una clara orientación preferente en relación con la concavidad de las conchas. A excepción de la estación de muestreo LRI-2, las demás se caracterizan por presentar un porcentaje relativamente bajo de fragmentos redondeados. Finalmente, esta agrupación presenta una buena distribución de frecuencias en los tamaños de bioclastos, predominando los fragmentos de la clase de tamaños más pequeña (Fig. 5).

La agrupación $\mathrm{C}$ contiene las estaciones de muestreo LRI-3, LRI-4 y LRI-5 (Fig. 3). Las dos primeras muestras coinciden con los dos niveles carbonatados que aparecen en la sección, mientas que la tercera se sitúa en las arenas con laminaciones en artesa que hay inmediatamente por encima de la Calcarenita I (Fig. 2). Por tanto, es una 


\begin{tabular}{|c|c|c|c|c|c|c|c|c|c|c|c|c|c|c|c|c|c|c|c|c|c|c|}
\hline \multirow[b]{2}{*}{ Estación } & \multirow[b]{2}{*}{$\mathrm{N}$} & \multirow{2}{*}{$\begin{array}{l}\text { Vol } \\
(\%)\end{array}$} & \multirow{2}{*}{ Fragm } & \multirow[b]{2}{*}{ Comp } & \multicolumn{3}{|c|}{ Tamaño } & \multicolumn{2}{|c|}{ Articula. } & \multicolumn{2}{|c|}{ Angulo } & \multicolumn{4}{|c|}{ Otientación } & \multicolumn{3}{|c|}{ Preserv. concha } & \multicolumn{2}{|c|}{ Aristas } & \multicolumn{2}{|c|}{ Interacciones } \\
\hline & & & & & Media & $\mathrm{sd}$ & $\operatorname{Max}$ & $\mathrm{Si}$ & No & Media & $\mathrm{sd}$ & Arriba & Abajo & Vertic & Plano & Orig. & Molde & $\operatorname{Re}$ & A. & R. & Perfor. & Encos. \\
\hline 1 & 0,004 & $<5$ & 87,5 & 12,5 & 1,5 & 1,4 & 5,0 & 0,0 & 100,0 & 28,3 & 30,4 & 43,8 & 31,3 & 12,5 & 12,5 & 100,0 & 0,0 & 0,0 & 100. & 0,0 & 0,0 & 0,0 \\
\hline 2 & 0,053 & \begin{tabular}{|l|}
$5-$ \\
10 \\
\end{tabular} & 98,1 & 1,9 & 0,8 & 0,3 & 1,8 & 0,0 & 100,0 & 45,8 & 34,1 & 21,1 & 18,9 & 21,1 & 38,9 & 100,0 & 0,0 & 0,0 & 25,7 & 74,3 & 0,0 & 0,0 \\
\hline 3 & 0,134 & \begin{tabular}{|l|}
$30-$ \\
60 \\
\end{tabular} & 98,6 & 1,4 & 1,1 & 0,6 & 5,7 & 0,0 & 100,0 & 21,7 & 24,8 & 30,9 & 19,1 & 2,6 & 47,4 & $100,0 \mid$ & 0,0 & 0,0 & 24,8 & 75,2 & 0,0 & 0.9 \\
\hline $3 a$ & 0,029 & $<5$ & 99,1 & 0,9 & 0,8 & 0,5 & 4,0 & 0,0 & 100,0 & 44,7 & 33,3 & 27,5 & 26,6 & 15,6 & 30,3 & 100,0 & 0,0 & 0,0 & 52,6 & 47,4 & 2,6 & 0,0 \\
\hline $3 b$ & 0,076 & \begin{tabular}{|l|}
$5-$ \\
15 \\
\end{tabular} & 94,7 & 5,3 & 1,1 & 0,7 & 5,5 & 0,0 & 100,0 & 46,1 & 30,6 & 26,6 & 21,1 & 12,8 & 39,4 & 100,0 & 0,0 & 0,0 & 44,9 & 55,1 & 2,6 & 1,7 \\
\hline $3 b-p$ & 0,200 & 40 & 94,0 & 6,0 & 1,1 & 0,6 & 3,0 & 0,0 & 100,0 & 51,4 & 30,3 & 35,1 & 21,6 & 17,5 & 25,8 & 100,0 & 0,0 & 0,0 & 76,0 & 24,0 & 9,0 & 0,0 \\
\hline 4 & 0,149 & \begin{tabular}{|l|}
$50-$ \\
55 \\
\end{tabular} & 96,5 & 3,5 & 1,2 & $1,0 \mid$ & 14,0 & 0,0 & 100,0 & 32,1 & 27,5 & 26,3 & 25,4 & 6,8 & 41,5 & 86,4 & 13,4 & 0,2 & 19,1 & 80,9 & 0,4 & 0,0 \\
\hline 5 & 0,152 & \begin{tabular}{|l}
$40-$ \\
55 \\
\end{tabular} & 97,9 & 2,1 & 1,0 & 0,6 & 4,7 & 0,0 & 100,0 & 31,4 & 28,5 & 18,4 & 12,5 & 7,5 & 61,6 & 100,0 & 0,0 & 0,0 & 21,5 & 78,5 & 0,3 & 0,8 \\
\hline 6 & 0,165 & $\begin{array}{r}40- \\
60 \\
\end{array}$ & 95,5 & 4,5 & 2,6 & 1,9 & 15,0 & 0,5 & 99,5 & 34,0 & 27,3 & 32,2 & 24,5 & 6,3 & 37,0 & 100,0 & 0,0 & 0,0 & 15,2 & 84,8 & 10,8 & 2,4 \\
\hline 7 & 0,082 & $\begin{array}{c}20 \\
40\end{array}$ & 95,7 & 4,3 & 2,1 & 1,6 & 11,2 & 0,0 & 100,0 & 33,9 & 25,5 & 31,4 & 22,2 & 2,6 & 43,8 & 100,0 & 0,0 & 0,0 & 22,0 & 78,0 & 5,1 & 3,3 \\
\hline
\end{tabular}

Tabla 1. Atributos tafonómicos medidos en la sección de Los Ranchos I. Todos los resultados están expresados en porcentaje, excepto N, calculado como el número de bioclastos por superficie de muestreo $\left(400 \mathrm{~cm}^{2}\right)$. Vol. = porcentaje de bioclastos por volumen de roca; Fragm. = fragmentación; Comp. = completos; sd = desviación estándar; Max. = máximo; Articula. = articulación; Vertic. = vertical; Presev. = preservación; Orig. = original; Re. = recristalización; A = aguda; R. = redondeada; Perfor. = perforaciones; Encos. = encostramientos.

Taphonomic attributes measured in the Los Ranchos I section. All values are in percentages, except $N$, which is calculated as the number of bioclasts per sampling area $\left(400 \mathrm{~cm}^{2}\right)$. Vol. $=$ percentage of bioclasts per rock volume; Fragm. = fragmentation $;$ Comp. = complete; $s d=$ standard deviation; Max. = largest shell size; Articula.$=$ articulation; Vertic $=$ vertical $;$ Preserv. = skeletal shell preservation; Orig. = original; Re. = recrystalized; A. $=$ sharp; $R .=$ rounded $;$ Perfor.$=$ borings; Encos. $=$ encrusting.

agrupación que engloba muestras con una litología y estructuras sedimentarias similares. Las propiedades tafonómicas que diferencian esta agrupación son (Fig. 6): a) clasificación de tamaños de bioclastos bimodal, dominando los fragmentos dentro de las dos primeras clases de tamaños; b) predominio de conchas dispuestas concordantemente; c) porcentaje de fósiles por volumen de roca relativamente alto; d) predominio de valvas planas; y, e) presentar los valores más bajos de interacciones. La estación LRI-4, cuya unión con las otras dos estaciones se sitúa a más distancia en el análisis cluster (Fig. 3), se diferencia de ellas porque es la única en la que se ha registrado la aparición de moldes (Fig. 6).

Por último, la agrupación D está formada por las estaciones LRI-6 y LRI-7 (Fig. 3), ambas situadas en la Calcarenita III (Fig. 2). De éstas, la primera incluye acumulaciones residuales de fósiles en la base de canales y en los surcos de los ripples, mientras que la segunda se localiza en la matriz carbonatada en la que se incluyen los canales y las artesas. Ambas estaciones de muestreo se separan de las demás por presentar las distribuciones de tamaño más dispersas y desplazadas hacia las clases de tamaños mayores, ya que en ellas están contenidas las conchas de mayor tamaño encontradas en la sección. Además, estas dos estaciones se caracterizan también por presentar los porcentajes más altos de interacciones y de fragmentos redondeados. Otra propiedad distintiva de esta agrupación es que muestra un dominio de conchas orientadas con ángulos inferiores a $30^{\circ}$ (Fig. 7).

\section{SECCIÓN DE LOS RANCHOS II}

\section{ESTRATIGRAFÍA Y ASOCIACIONES DE FÓSILES}

La sección de Los Ranchos II (Fig. 2) es la más septentrional de las dos estudiadas. En la base vuelve a reconocerse el contacto discordante entre la unidad pliocena estudiada y las margas post-evaporíticas del Messiniense. Este contacto está representado por una superficie bioturbada, con las galerías excavadas en las margas miocenas y rellenas por los limos-arenas del Plioceno.

Al igual que ocurría en la sección de Los Ranchos I, aquí también se diferencian dos tramos, uno arenoso y otro donde se intercalan los paquetes carbonatados (Fig. 2). El primer tramo comienza con unos limos-arenas finas masivas de color grisáceo-amarillento. Como en la sección anterior, se observa un moteado de bioturbación (ii2-ii3) e intervalos intensamente bioturbados. La fauna que se encuentra en los primeros metros de la sección está representada por Korobkovia, Chlamys, Pecten, Neopycnodonte cochlear, Neopycnodonte navicularis y el gasterópodo Scalaria, junto con algunos moldes de corales solitarios tipo Flabellum.

Las arenas cambian a más micáceas según se sube en la serie, y se observa la presencia de cantos de areniscas cementadas, cantos de cuarcita y cantos carbonatados (fundamentalmente dolomías). Asimismo, se produce un aumento de fragmentos de conchas. La asociación 


\begin{tabular}{|c|c|c|c|c|c|c|c|c|c|c|c|c|c|c|c|c|c|c|c|c|c|c|}
\hline \multirow[b]{2}{*}{ Estación } & \multirow[b]{2}{*}{ N } & \multirow{2}{*}{$\begin{array}{l}\text { Vol } \\
(\%)\end{array}$} & \multirow{2}{*}{ Fragm } & \multirow[b]{2}{*}{ Comp } & \multicolumn{3}{|c|}{ Tamaño } & \multicolumn{2}{|c|}{ Articula. } & \multicolumn{2}{|c|}{ Angulo } & \multicolumn{4}{|c|}{ Orientación } & \multicolumn{3}{|c|}{ Preserv. concha } & \multicolumn{2}{|c|}{ Aristas } & \multicolumn{2}{|c|}{ Interacciones } \\
\hline & & & & & Media & $s d$ & $\operatorname{Max}$ & $\mathrm{Si}$ & No & Media & sd & Arriba & Abajo & Vertic & Plano & Orig. & Molde & $\mathrm{Re}$ & A. & R. & Perfor. & Encos. \\
\hline 1 & 0,023 & 5 & 95,7 & 4,3 & 0,8 & 0,6 & 5,5 & 0,0 & 100,0 & 42,0 & 32,5 & 19,3 & 17,0 & 14,8 & 48,9 & 100 & 0,0 & 0,0 & 47,3 & 52,7 & 0,0 & 0 \\
\hline 2 & 0,028 & $<5$ & 91,2 & 8,8 & 0,8 & 0,4 & 3,0 & 0,0 & 100,0 & 43,5 & 33.6 & 22,9 & 21,1 & 15,6 & 40,4 & 100 & 0,0 & 0,0 & 24,8 & 75,2 & 0,0 & 0,9 \\
\hline 2-pl & 0,183 & 55 & 90,9 & 9,1 & 1,4 & 1,1 & 6,9 & 0,0 & 100,0 & 39,9 & 32,8 & 39,6 & 15,1 & 13,2 & 32,1 & 100 & 0,0 & 0,0 & 30,9 & 69,1 & 1,8 & 1,8 \\
\hline $2-p 2$ & 0,157 & 55 & 83,0 & 17,0 & 1,2 & 0,6 & 2,9 & 0,0 & 100,0 & 40,9 & 30,3 & 44,7 & 19,1 & 10,6 & 25,5 & 100 & 0,0 & 0,0 & 31,9 & 68,1 & 2,1 & 4,3 \\
\hline 3 & 0,136 & 27 & 96,6 & 3,4 & 1,4 & 0,7 & 5,0 & 0,6 & 99,4 & 44,3 & 30.7 & 51,6 & 20,6 & 11,0 & 16,8 & 100 & 0,0 & 0,0 & 56,2 & 43,8 & 6,6 & 3,1 \\
\hline 4 & 0,028 & $<5$ & 86,4 & 13,6 & 0,9 & 0,6 & 5,5 & 4,7 & 95,3 & 48,3 & 32,5 & 25,7 & 15,8 & 16,8 & 41,6 & 100 & 0,0 & 0,0 & 21,8 & 78,2 & 1,8 & 0,9 \\
\hline 4-pl & 0,219 & 55 & 68,0 & 32,0 & 1,4 & 0,6 & 3,3 & 0,0 & 100,0 & 51,6 & 28,4 & 44,0 & 28,0 & 12,0 & 16,0 & 100 & 0,0 & 0,0 & 48,0 & 52,0 & 4,0 & 10,0 \\
\hline 4-p2 & 0,118 & 55 & 66,7 & 33,3 & 1,7 & 0,7 & 3,4 & 11,5 & 88,5 & 40,6 & 30,9 & 54,2 & 33,3 & 8,3 & 4,2 & 100 & 0,0 & 0,0 & 48,1 & 51,9 & 11,1 & 11,1 \\
\hline 4-p3 & 123 & 53 & 4,3 & 35,7 & 1,6 & 0,7 & 3,5 & 0,0 & 100,0 & 50,4 & 32,2 & 37,0 & 37,0 & 22,2 & 3,7 & 100 & 0,0 & 0,0 & 25,0 & 75,0 & 25,0 & 14,3 \\
\hline 5 & 0,118 & 55 & 95,1 & 4,9 & 1,0 & 0,5 & 4,7 & 0,0 & 100,0 & 22,0 & 22,1 & 39,7 & 22,2 & 2,9 & 35,2 & 75,5 & 24,5 & 0,0 & 13,2 & 86,8 & 0,3 & 0,3 \\
\hline 6 & 0,123 & 50 & 96,1 & 3,9 & 1,1 & 0,7 & 10,0 & 2,2 & 97,8 & 25,3 & 25,6 & 31,1 & 26,8 & 3,6 & 38,4 & 80,0 & 20,0 & 0,0 & 1,0 & 99,0 & 0,0 & 1,0 \\
\hline 7 & 0,205 & 35 & 94,3 & 5,7 & 0,8 & 0,4 & 5,8 & 0,1 & 99,9 & 49,9 & 29,0 & 35,2 & 24,0 & 13,4 & 27,5 & 100 & 0,0 & 0,0 & 94,4 & 5,6 & $\overline{0,6}$ & 0,4 \\
\hline 8 & 0,167 & 60 & 94,7 & 5,3 & 1,3 & 1,3 & 13,0 & 1,4 & 98,6 & 29,2 & 27,9 & 34,8 & 23,9 & 7,3 & 34,0 & 92,2 & 7,8 & 0,0 & 11,9 & 88,1 & 2,5 & 0,9 \\
\hline 9 & 0,130 & 50 & 93,4 & 6,6 & 2,1 & 1,7 & 8,5 & 0,0 & 100,0 & 29,1 & 23,7 & 32,7 & 22,0 & 0,8 & 44,5 & 87,6 & 12,4 & 0,0 & 7,6 & 92,4 & 3,9 & 1,5 \\
\hline
\end{tabular}

Tabla 2. Atributos tafonómicos medidos en la sección de Los Ranchos II. Abreviaturas como en la Tabla 1.

Taphonomic attributes measured in the Los Ranchos II section. Values and abbreviations as in Table 1.

faunística que caracteriza esta zona del primer tramo está dominada por Chlamys, Neopycnodonte, Ostrea edulis, Pecten de gran tamaño, equinodermos, briozoos (entre ellos se encuentran colonias esféricas, colonias foliosas, colonias de crecimiento erecto con ramas delicadas [vinculariiforme] y de crecimiento erecto con ramas gruesas [adeoniforme]), balánidos, braquiópodos y el gasterópodo Scalaria. Los fósiles se encuentran dispersos en el sedimento o en pequeñas concentraciones aisladas (principalmente de Neopycnodonte).

A continuación, intercalado en las arenas se observa el nivel de rodolitos equivalente al de la sección Los Ranchos I, con un espesor de $15 \mathrm{~cm}$. Asociados a las algas aparecen Ostrea, Spondylus, Neopycnodonte, Gigantopecten, Pecten, Chlamys, Clypeaster y pequeños equinodermos regulares. Destaca la presencia de algunos fragmentos de huesos de mamíferos marinos. Muy ocasionalmente se encuentran moldes de corales solitarios.

Hacia la mitad del primer tramo se intercala un nivel de acumulación de conchas que muestra una gradación positiva en el tamaño de los bioclastos y un contacto neto a la base y gradual a techo (Fig. 2). En determinados puntos, la base de esta acumulación penetra en las arenas infrayacentes por medio de galerías atribuidas a madrigueras que pueden alcanzar tamaños de hasta $50 \mathrm{~cm}$ de longitud. Este nivel está formado casi exclusivamente por Neopycnodonte cochlear, algunos de los cuales se pueden encontrar desarticulados pero cementados unos sobre otros. Además, contiene otros ostreidos, pectínidos (entre ellos Chlamys y Flexopecten flexuosa), Spondylus, equinodermos, Ditrupa, briozoos y braquiópodos.
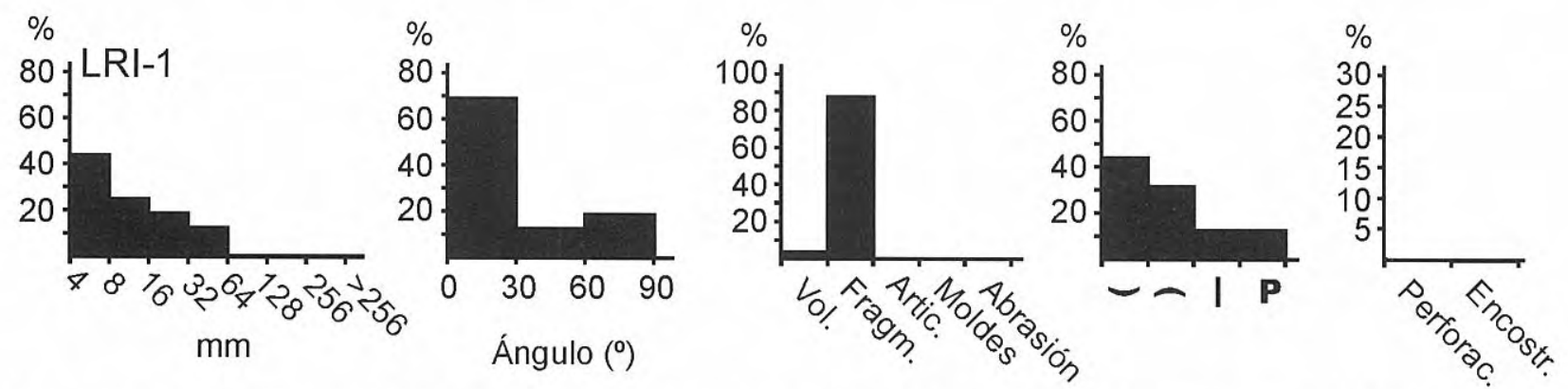

Figura 4. Histogramas de frecuencia de tamaños, inclinación de los bioclastos (biofábrica) y otras propiedades tafonómicas que caracterizan la agrupación A en la sección de Los Ranchos I. Vol. = volumen; Fragm. = fragmentación; Artic. = articulación; Perforac. $=$ perforaciones; Ecostr. $=$ encostramientos.

Histograms of the size-sorting, inclination of bioclasts (biofabric), and other taphonomic attributes characterising group $A$ of the Los Ranchos I section. Vol. = volume; Fragm. = fragmentation; Artic. = articulation; Perforac. $=$ borings; Ecostr. = encrusting. 

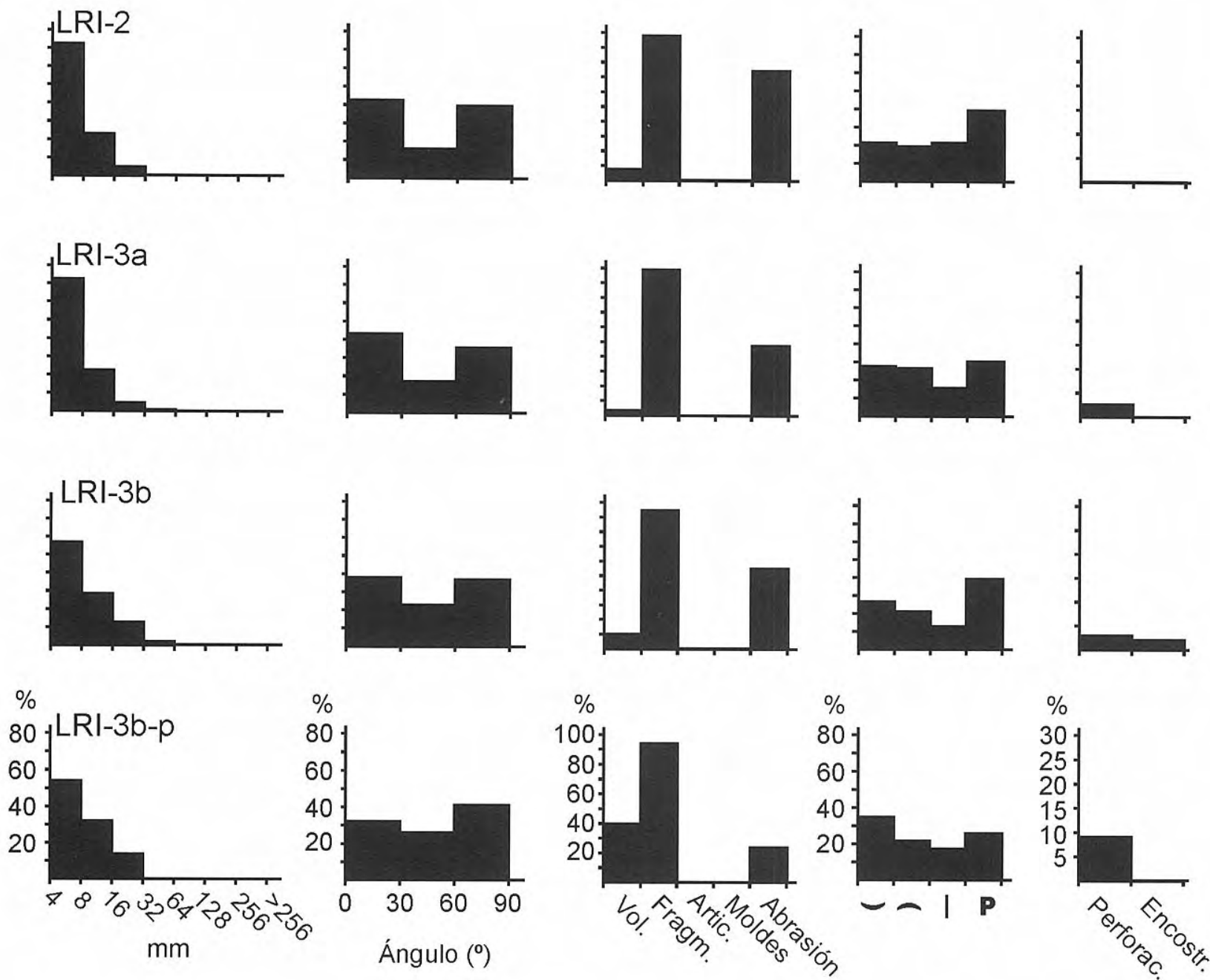

Figura 5. Histogramas de frecuencia de tamaños, inclinación de los bioclastos (biofábrica) y otras propiedades tafonómicas que caracterizan la agrupación B en la sección de Los Ranchos I. Abreviaturas como en la Fig. 4.

Histograms of the size-sorting, inclination of bioclasts (biofabric), and other taphonomic attributes characterising group $B$ of the Los Ranchos I section. Abbreviations like in Fig. 4.

Al final del primer tramo se intercala un paquete de calcarenitas-calcirruditas con gran cantidad de cantos de cuarcitas, y cuyo contacto inferior es gradual (Fig. 2). Dicho paquete transiciona lateralmente hacia el $\mathrm{S}$ a arenas bioclásticas cementadas y, finalmente, a arenas masivas. Internamente se observan laminaciones cruzadas en artesa de poca amplitud y muy tendidas, orientadas hacia el $\mathrm{N}$, y una cierta gradación negativa en el tamaño de los bioclastos. La asociación de fósiles que caracteriza este nivel está representada por diferentes tipos de bivalvos, como Neopycnodonte, Spondylus, Chlamys y otros pectínidos, briozoos y equinodermos. También se han observado Ditrupa, balánidos, corales solitarios y numerosos moldes de Turritella. Es destacable la gran cantidad de serpúlidos (Ditrupa) presentes en el sedimento y de algas coralináceas, que representan aproximadamente el $30-40 \%$ del volumen de la roca. Estas aparecen como fragmentos de ramas o formando pequeños rodolitos.

El primer tramo de la serie culmina con el primer paquete calcarenítico, Calcarenita I, que alcanza hasta 3 $\mathrm{m}$ de potencia (Fig. 2). Se trata de calcarenitas y calcirruditas muy bioclásticas y cementadas con laminaciones cruzadas en artesa con un sentido de migración hacia el N. En la base del paquete, se reconocen concentraciones residuales de conchas en los surcos de las artesas y en la base de canales. En estas acumulaciones hay gran cantidad de fragmentos inidentificables de bivalvos, muchos de ellos conservados como moldes. Se han reconocido pectínidos, ostreidos, Neopycnodonte, Isognomon, Spondylus, gasterópodos, moldes de corales solitarios, briozoos (que aparecen en alta proporción), equinodermos y serpúlidos como Ditrupa. La concentración de algas es de aproximadamente el 40-50\%.

El segundo tramo comienza con unas arenas de grano medio con cantos dispersos y concentraciones en vaina de bioclastos. Aparecen equinodermos, como Clypeaster y cidáridos, y huesos de mamíferos marinos dispersos en el sedimento. La concentración de algas es superior al $50 \%$, y se observan rodolitos de hasta $5 \mathrm{~cm}$. También se encuentran inmersos en el sedimento grandes ejemplares de Ostrea lamellosa, de hasta $16 \mathrm{~cm}$ de tamaño, muy perforados. 

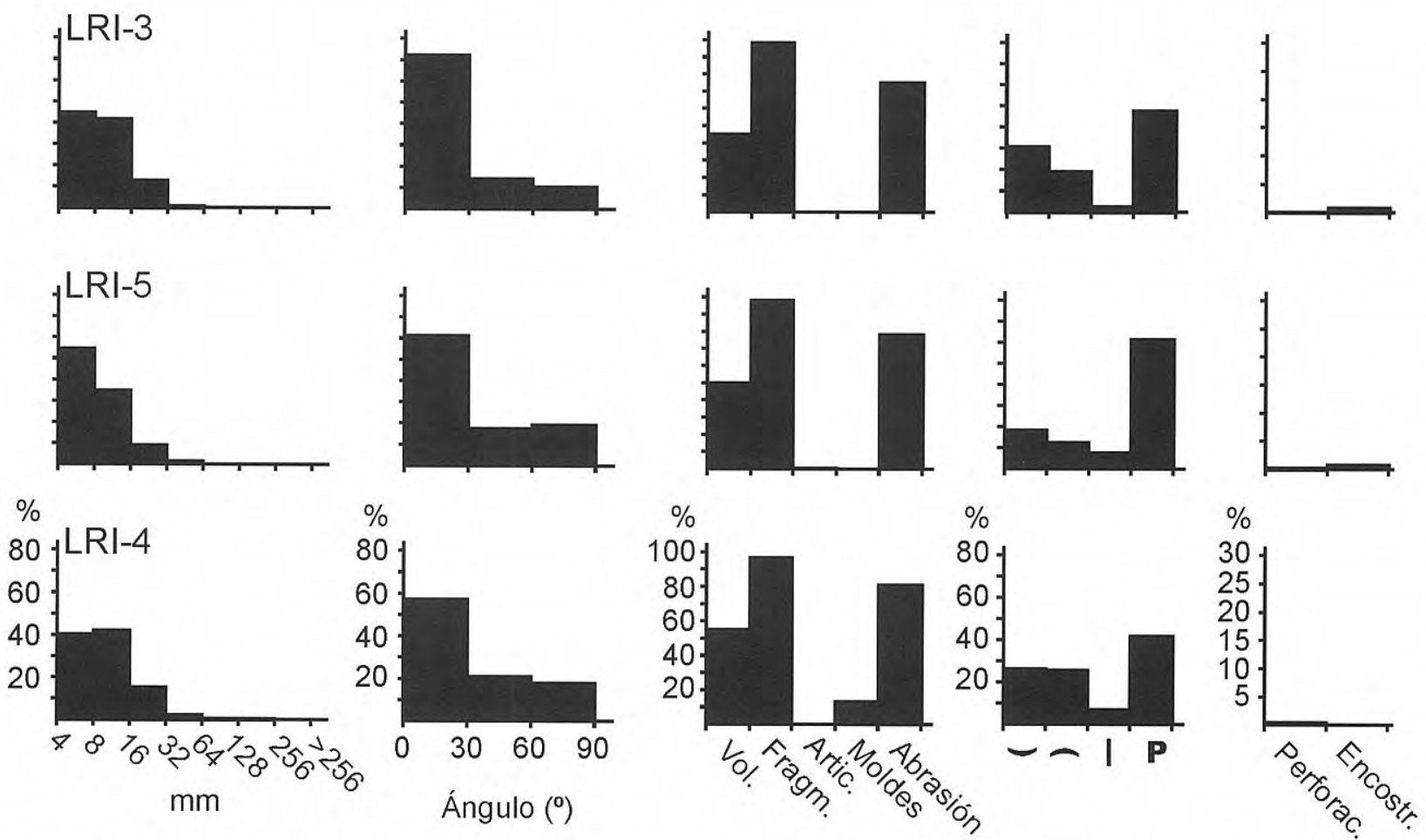

Figura 6. Histogramas de frecuencia de tamaños, inclinación de los bioclastos (biofábrica) y otras propiedades tafonómicas que caracterizan la agrupación C en la sección de Los Ranchos I. Abreviaturas como en la Fig. 4.

Histograms of the size-sorting, inclination of bioclasts (biofabric), and other taphonomic attributes characterising group $C$ of the Los Ranchos I section. Abbreviations like in Fig. 4.

Hacia techo se pasa gradualmente a unas arenas muy bioclásticas, formadas casi exclusivamente por conchas de bivalvos de muy pequeño tamaño. Se observan acumulaciones fosilíferas con geometrías tubulares o de embudo muy manifiestas, que llegan a alcanzar dimensiones de hasta $1 \mathrm{~m}$ de longitud (Fig. 8). Los fósiles dentro de estas acumulaciones presentan un ordenamiento similar; aparecen las conchas dispuestas horizontalmente o con bajo ángulo en los márgenes circundantes a la apertura y, según se adentran en la madriguera, aumenta la inclinación de forma que se disponen paralelamente a los bordes de la misma. Finalmente, las conchas confluyen en el fondo adaptándose a la forma de éste (Fig. 8). Es decir, la inclinación de las propias conchas delimita la acumulación. La superposición de todas estas concentraciones bioclásticas da lugar a una acumulación homogénea que refleja una bioturbación de fondo en la que se diferencian algunas madrigueras discretas. La asociación fosilífera que caracteriza estas arenas está casi exclusivamente dominada por formas juveniles de bivalvos. Se han reconocido mitílidos (mayoritariamente Modiolus), Anomia, Amusium cristatum, Chlamys, Neopycnodonte, otros pectínidos y ostreidos, además de equinodermos, briozoos, balánidos y serpúlidos. También aparecen dispersos restos de algas coralináceas.

Por encima de estas arenas aparece la Calcarenita II (Fig. 2) con laminaciones cruzada en artesa de estructuras que migraban hacia el N. Aunque el muro de estas arenas es gradual, aparece como erosivo o neto debido a la existencia de una cementación diferencial. Como en la sección Los Ranchos I, hay acumulaciones residuales de conchas en la base de canales y en los surcos de las artesas que muestran una gradación positiva. La asociación faunística está formada por pectínidos, ostreidos (entre ellos Neopycnodonte), briozoos, serpúlidos, gasterópodos y balánidos. La concentración de algas oscila entre el 10 y el $20 \%$, aunque en algunas zonas puede superar el $70 \%$.

En el techo se observan cuerpos canaliformes amalgamados que erosionan los materiales infrayacentes. Estos cuerpos corresponden al último paquete calcarenítico observado en Los Ranchos I, la Calcarenita III (Fig. 2). El tramo arenoso que queda entre los dos últimos paquetes carbonatados se acuña hacia el $\mathrm{N}$, de forma que las Calcarenitas II y III se amalgaman dando un único cuerpo calcáreo.

\section{RESULTADOS TAFONÓMICOS}

En la serie de Los Ranchos II se han establecido 14 estaciones de muestreo para el estudio tafonómico. Los resultados obtenidos se muestran en la Tabla 2. A partir de los datos numéricos recogidos en dicha tabla, un análisis cluster en modo $\mathrm{Q}$ permite diferenciar tres agrupaciones de muestras (Fig. 9). La agrupación A comprende las estaciones de muestreo situadas en arenas (Figs. 2 y 9). Esta agrupación puede dividirse en dos subagrupaciones. Una de ellas, subagrupación $\mathrm{A}_{1}$, incorpora todas las estaciones de muestreo situadas en arenas masivas (LRII-1, LRII-2 y LRII-4). La otra, 

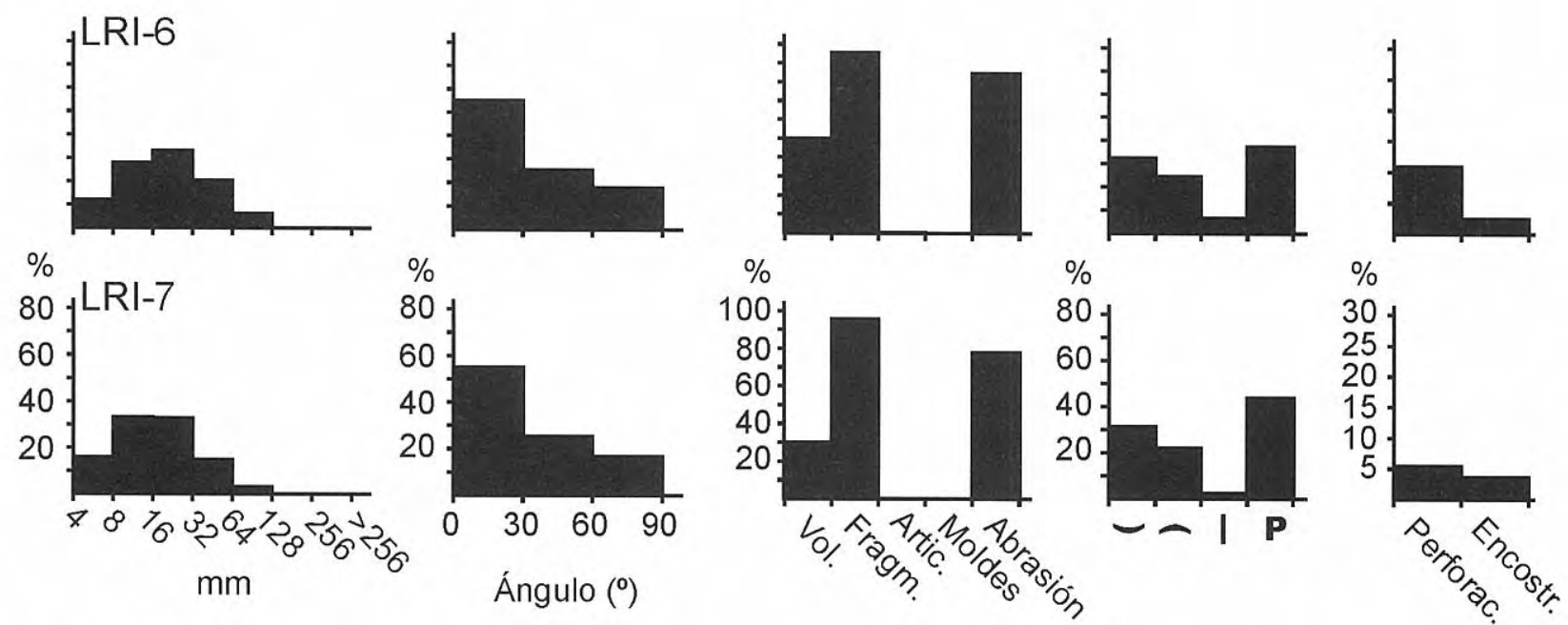

Figura 7. Histogramas de frecuencia de tamaños, inclinación de los bioclastos (biofábrica) y otras propiedades tafónomicas que caracterizan la agrupación D en la sección de Los Ranchos I. Abreviaturas como en la Fig. 4.

Histograms of the size-sorting, inclination of bioclasts (biofabric), and other taphonomic attributes characterising group D of the Los Ranchos I section. Abbreviations like in Fig. 4.

subagrupación $\mathrm{A}_{2}$, engloba las acumulaciones en vaina (LRII-2-p1, LRII-2-p2 y LRII-4-p1) y las concentraciones de fósiles modificadas por la actividad bioturbadora de otros organismos (LRII-3, y LRII-7).

La subagrupación $A_{1}$ se caracteriza porque los fósiles están prácticamente ausentes, dando unos porcentajes de bioclastos por volumen de roca muy bajos $(0,023$ a 0,028 bioclastos $/ \mathrm{cm}^{2}$ ) y un empaquetamiento muy disperso ( $\leq$ $5 \%$ ) (Tabla 2). En esta subagrupación se incluyen las estaciones de muestreo con el tamaño medio de los bioclastos más pequeño. Como consecuencia, se caracterizan por tener una distribución de tamaños marcadamente unimodal, con claro predominio de la clase de tamaños más pequeña. El pequeño tamaño de los bioclastos también explica el hecho de que dominen los fragmentos planos. Con respecto a la orientación, se observa una distribución bimodal, con la mayoría de los fósiles orientados concordante o perpendicularmente (Fig. 10).

El subgrupo $A_{2}$ se diferencia del anterior por mostrar una gran abundancia de conchas por volumen de roca $y$, por tanto, por un empaquetamiento denso (Tabla 2). El rasgo tafonómico más significativo de esta subagrupación es el alto porcentaje de fragmentos que presentan las aristas agudas, lo que implica un bajo grado de abrasión (Fig. 11). Además, hay un mayor porcentaje de conchas orientadas con la concavidad hacia arriba, hay una gran dispersión de tamaños de bioclastos, y el porcentaje de interacciones es relativamente alto (Fig. 11). Hay que destacar que la estación de muestreo LRII-7 es la que se une a este subgrupo a mayor distancia en el cluster (Fig. 9). Esto se debe a que la media de tamaños y el porcentaje de interacciones son inferiores a los del resto de la asociación. No obstante, la propiedad tafonómica distintiva de esta estación de muestreo es que casi el 100\% de los fragmentos muestran las aristas agudas (Fig. 11).

La agrupación B comprende las estaciones de muestreo LRII-5, LRII-6, LRII-8 y LRII-9, todas ellas correspondientes a calcarenitas (Figs. 2 y 9). Esta agrupación se caracteriza por el alto porcentaje de bioclastos por volumen de roca $(\sim 30 \%)$ (Tabla 2$)$. Además, las estaciones de muestreo incluidas en esta agrupación son las únicas que presentan moldes (Fig. 12). Así mismo, muestran una alta dispersión en los tamaños de bioclastos. En relación con la orientación de las conchas, dominan aquellas dispuestas con ángulos inferiores a $30^{\circ}$. Esto hace que en esta agrupación se den los valores medios de inclinaciones más bajos (Tabla 2). Los fragmentos planos son los más abundantes, aunque entre los que presentan una curvatura detectable, abundan los que se disponen con la concavidad hacia arriba. Finalmente, otro rasgo tafonómico distintivo de esta agrupación es la altísima proporción de fragmentos con las aristas redondeadas, lo que denota un alto grado de abrasión (Fig. 12).

$\mathrm{Al}$ igual que con la agrupación anterior, la agrupación B puede dividirse en dos subgrupos (Fig. 9), la subagrupacion $\mathrm{B}_{1}$, que incluye las muestras localizadas en la matriz calcarenítica (LRII-5 y LRII-6), y la subagrupacion $\mathrm{B}_{2}$, que engloba las concentraciones residuales de fósiles de las bases de canales y de surcos de artesas (LRII-8 y LRII-9). La principal diferencia entre ambas subagrupaciones es que la segunda muestra un mayor grado de empaquetamiento e interacciones bióticas (Tabla 2, Fig. 12).

La última agrupación distinguida por el análisis cluster es la agrupación $\mathrm{C}$, en la que quedan dos acumulaciones en vaina (LRII-4-p2 y LRII-4-p3). Estas dos acumulaciones presentan unas características muy dispares entre sí, y esto se ve reflejado en el dendrograma, ya que su unión se produce a gran distancia (Fig. 9). Se caracterizan, sobre todo, por tener la proporción más alta de conchas completas, el porcentaje más bajo de conchas planas y la máxima proporción de conchas perforadas y con encostramientos (Fig. 13). 


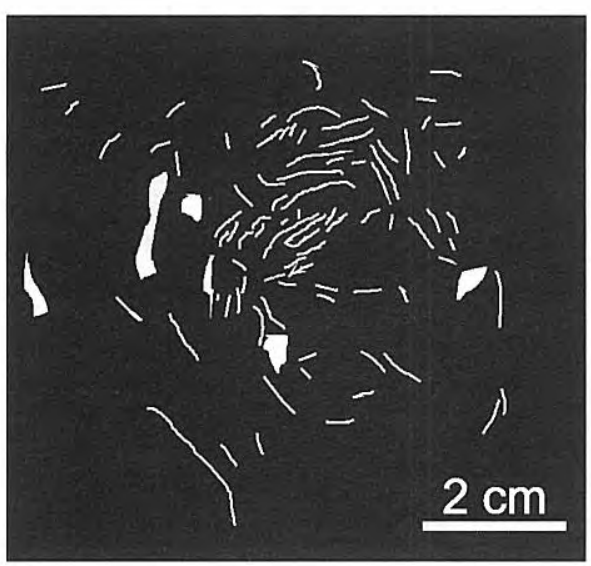

A

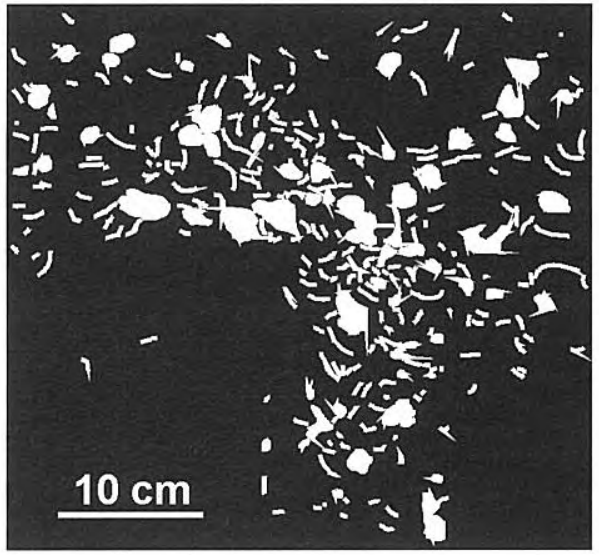

B

Figura 8. Corte transversal (A) y longitudinal (B) de rellenos de madrigueras. Obsérvese como se disponen las conchas paralelamente a los bordes de la madriguera en sección transversal. (Esquemas realizados a partir de fotos de campo.)

Transversal (A) and longitudinal (B) sections of filled burrows with bioclasts. Note that shells arrange parallel to the walls of the trace fossil in transverse section. (Sketch are made from field pictures.)

\section{DISCUSIÓN}

\section{INTERPRETACIÓN PALEOAMBIENTAL}

El carácter limoso y masivo de los sedimentos de la base del primer tramo, junto con la ausencia de estructuras sedimentarias, sugieren un medio sedimentario de baja energía. Estos materiales están dominados por Korobkovia, Amusium y Neopycnodonte, organismos adaptados a medios profundos y poco energéticos (Gould, 1971; Carter, 1972; Stanley, 1972, 1988; Hickman, 1984; Pérès, 1989; Poppe y Goto, 1993).

Desde un punto de vista tafonómico se observa un porcentaje de conchas completas bastante alto y la totalidad de dichas conchas conservan las aristas agudas o muestran una buena preservación de la ornamentación. Estos atributos tafonómicos son congruentes con un medio profundo de muy baja energía (Brett y Baird, 1986; Kidwell y Bosence, 1991; Parsons y Brett, 1991). La escasa abundancia de bioclastos y la total ausencia de interacciones de otros organismos sobre las conchas, indican que la tasa de sedimentación debió ser alta (Brett y Baird, 1986; Speyer y Brett, 1986, 1988; Kidwell y Bosence, 1991), de modo que el tiempo de exposición en la zona tafonómicamente activa (TAZ sensu Powell et al., 1989) no fue lo suficientemente importante, inhibiéndose así la acción de procesos bioestratinómicos destructivos (Speyer y Brett, 1988; Kidwell y Bosence, 1991; Parsons y Brett, 1991).

El predominio de las conchas orientadas con la concavidad hacia arriba se corresponde con el patrón observado por Emery (1968) y Salazar-Jiménez et al. (1982) para sedimentos de plataforma intensamente bioturbados. Esto coincide con el hecho de que en la base de la unidad estudiada la bioturbación puede ser intensa, con un índice de icnofábrica alto (ii4). Dicho patrón también se asemeja al observado por Callender et al. (1990) en sedimentos de plataforma por debajo del nivel del oleaje de tormentas. En conclusión, se puede inferir que los limos y arenas finas de la base de la secuencia pliocena estudiada se formaron en un medio de plataforma externa.

Según se sube en la serie las arenas se hacen más micáceas y aparecen cantos aislados de diferente naturaleza. Como se describió en los apartados previos,

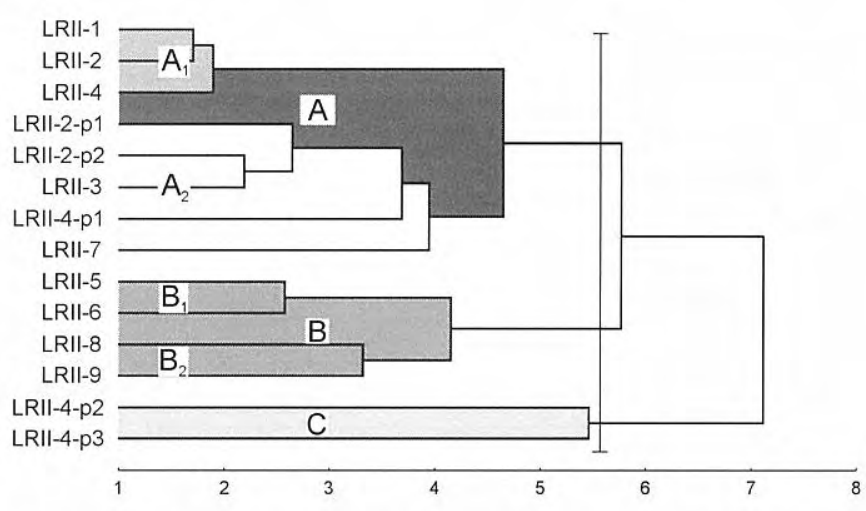

Figura 9. Análisis cluster de las propiedades tafonómicas medidas en la sección Los Ranchos II. Dendrograma obtenido a partir de un análisis cluster tipo Q usando el método del promedio simple y distancias euclídeas. La línea vertical marca la distancia más adecuada a la que se han realizado las agrupaciones según los métodos de Beale y Calinski.

Cluster analysis of the taphonomic attributes measured in Los Ranchos II section. Dendrogram obtained from a $Q$-mode cluster analysis, using unweigthted pair-group average method and Euclidean distances. The vertical line marks the most adequate distance to produce the groups according to the Beale and Calinski methods. 

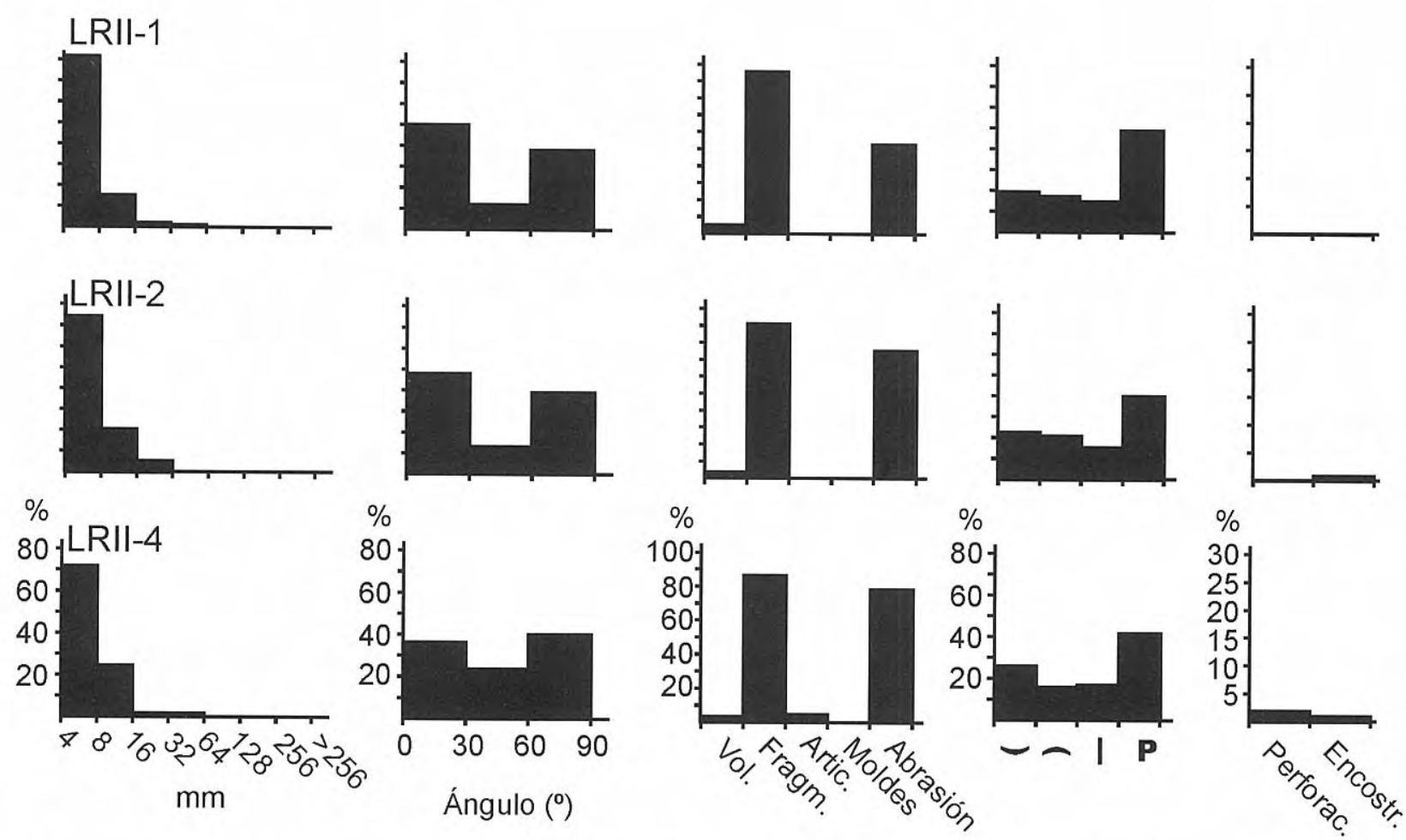

Figura 10. Histogramas de frecuencia de tamaños, inclinación de los bioclastos (biofábrica) y otras propiedades tafónomicas que caracterizan la agrupación $\mathrm{A}_{1}$ en la sección de Los Ranchos II. Abreviaturas como en la Fig. 4.

Histograms of the size-sorting, inclination of bioclasts (biofabric), and other taphonomic attributes characterising group A, of the Los Ranchos II section. Abbreviations like in Fig. 4.

los cantos pasan de ser casi exclusivamente volcánicos en los primeros metros de la secuencia a ser sustituidos por cantos metamórficos. Esto indica un cambio en la fuente de alimentación, de forma que en los primeros momentos de sedimentación pliocena el área fuente se localizaba en el basamento volcánico de Sierra de Gata y después pasó a tomar importancia Sierra Cabrera. Esto confirma el hecho de que durante el Plioceno Inferior, Sierra Cabrera debió comportarse como un relieve emergente que favoreció el desarrollo de potentes depósitos deltaicos al S de El Argamasón (Aguirre, 1998).

Hacia la mitad del primer tramo continúan las arenas, con propiedades tafonómicas muy parecidas lo que permite inferir condiciones ambientales muy similares. Esto lo corrobora el predominio de Neopycnodonte cochlear, bivalvo epifáunico que vive actualmente en el Mediterráneo en aguas profundas (entre 45 y $250 \mathrm{~m}$ ) (Pérès, 1989; Poppe y Goto, 1993). No obstante, se observa un considerable aumento en el contenido faunístico. Entre los pectínidos se produce un substancial incremento en la proporción de formas con costillas fuertes y con un fuerte grado de curvatura. Estos rasgos morfológicos se relacionan con ambientes más someros (Aguirre et al., 1996), a diferencia de los pectínidos con conchas lisas y sin ornamentación, aplanadas y con perímetros circulares (Gould, 1971; Stanley, 1972, 1988).

En cuanto a las propiedades tafonómicas, se observan algunas diferencias con relación a la base, que merecen ser mencionadas. El elevado grado de fragmentación y de desarticulación observado en esta parte de la unidad se atribuye a la acción de los organismos bioturbadores
(Brett y Baird, 1986; Speyer y Brett, 1988; Powell et al., 1989; Callender et al., 1990; Kidwell y Bosence, 1991; Parsons y Brett, 1991; Cadée, 1992, 1994a, 1994b; Best y Kidwell, 2000). De igual forma, las concavidades de las conchas muestran una distribución aleatoria. Este patrón de concavidades es muy característico de fondos sometidos a una intensa actividad de organismos bioturbadores (Emery, 1968; Clifton y Boggs, 1970; Clifton, 1971; Salazar-Jiménez et al., 1982; Speyer y Brett, 1986, 1988; Fürsich y Pandey, 1999). Por otro lado, el porcentaje de conchas que muestran buena preservación de la ornamentación y de las aristas desciende visiblemente. Estos atributos tafonómicos, junto a la presencia de algas coralináceas, indicarían que la tasa de sedimentación debió ser más baja. Sin embargo, las conchas no debieron permanecer demasiado tiempo expuestas sobre la superficie del sedimento dado que no se ha encontrado ningún tipo de interacción sobre ellas.

El nivel de algas coralináceas se caracteriza por contener también una gran abundancia de fragmentos de huesos de mamíferos marinos. Como se acaba de comentar, la alta concentración de algas sugiere una parada relativa en la sedimentación, que permitió la proliferación de las algas, las cuales requieren un medio con una baja tasa de sedimentación y escaso sedimento en suspensión (Milliman, 1977; Studencki, 1979; Bosence, 1983; Prager, 1987). La relativa abundancia de restos de mamíferos marinos en este nivel hace que presente una cierta analogía con las capas de huesos de la Formación Purísima (Plioceno) del centro de California descritas por Norris (1986). 

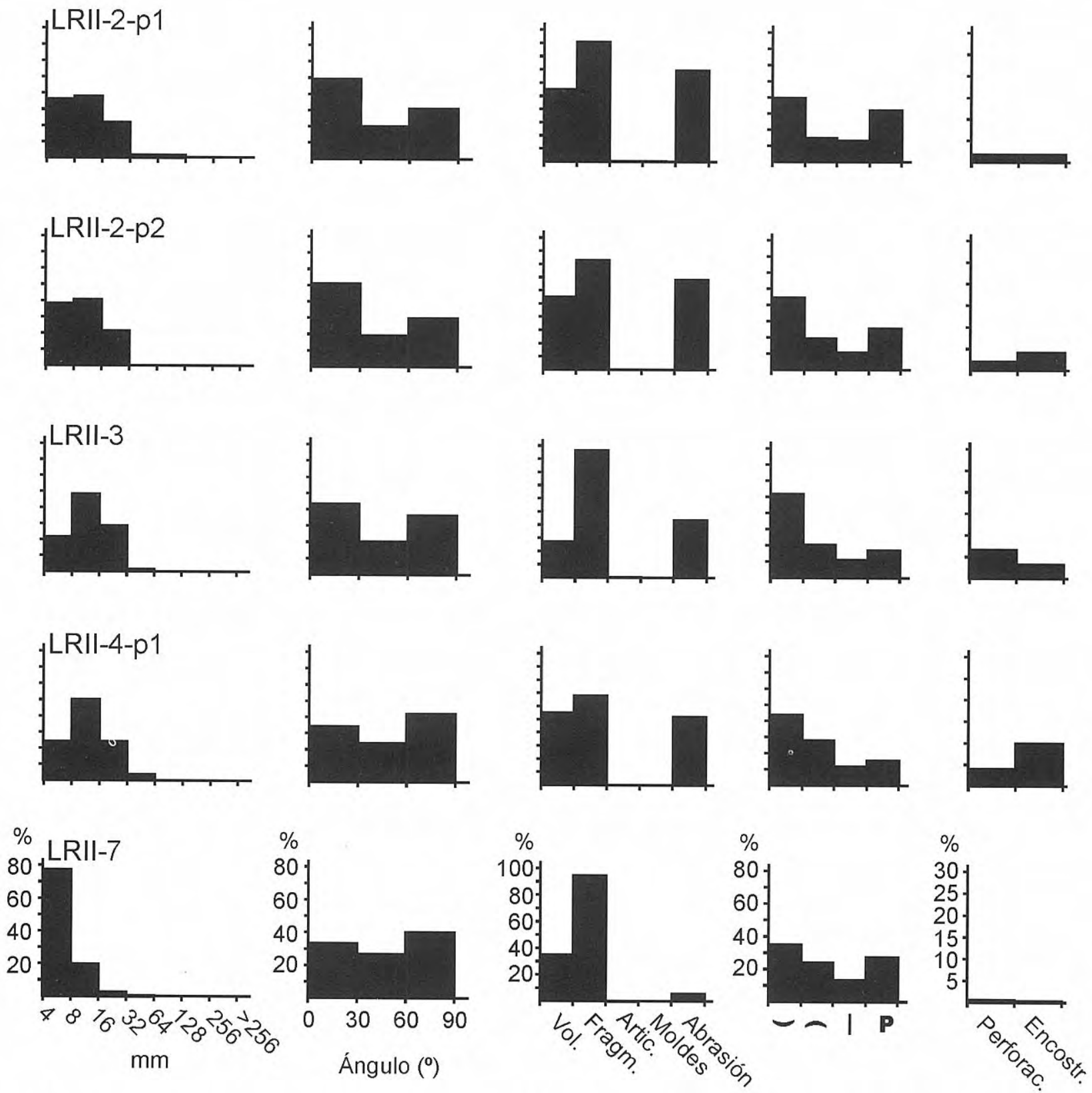

Figura 11. Histogramas de frecuencia de tamaños, inclinación de los bioclastos (biofábrica) y otras propiedades tafónomicas que caracterizan la agrupación $\mathrm{A}_{2}$ en la sección de Los Ranchos II. Abreviaturas como en la Fig. 4.

Histograms of the size-sorting, inclination of bioclasts (biofabric), and other taphonomic attributes characterising group $A_{2}$ of the Los Ranchos II section. Abbreviations like in Fig. 4.

Por encima del nivel de rodolitos en Los Ranchos I aparecen laminaciones cruzadas en artesa, que se hacen más marcadas y frecuentes a medida que se avanza hacia el S. En esta misma dirección, el substrato mioceno sobre el que se depositó el conjunto plioceno estudiado asciende topográficamente. Por el contrario, hacia el N, hacia Los Ranchos II, la cota topográfica del contacto Mioceno/Plioceno desciende. En esta misma dirección, la estratificación cruzada desaparece, pasándose a arenas masivas. Todo esto sugiere una somerización relativa hacia el S y una profundización hacia el N. Dicha profundización está corroborada por la asociación de fósiles que caracteriza las arenas masivas (Neopycnodonte, Amusium y Ditrupa) por encima del nivel de rodolitos en Los Ranchos II. Ditrupa, por ejemplo, forma en la actualidad concentraciones densas monotípicas en zonas de la plataforma externa (Ten Hove y Smith, 1990; Ten Hove y Den Hurk, 1993).

De igual forma, unos metros por encima del nivel de rodolitos en Los Ranchos II se encuentra el nivel de Neopycnodonte. Este nivel constituye una acumulación de tipo III en el sentido de (Kidwell, 1985, 1986). Según esta autora este tipo de acumulaciones se forman con una tasa de sedimentación inicialmente muy lenta, responsable de una alta concentración de restos fósiles, que aumenta progresivamente. La acumulación está formada casi exclusivamente por Neopycnodonte cochlear. La alta fragmentación y desarticulación, así 

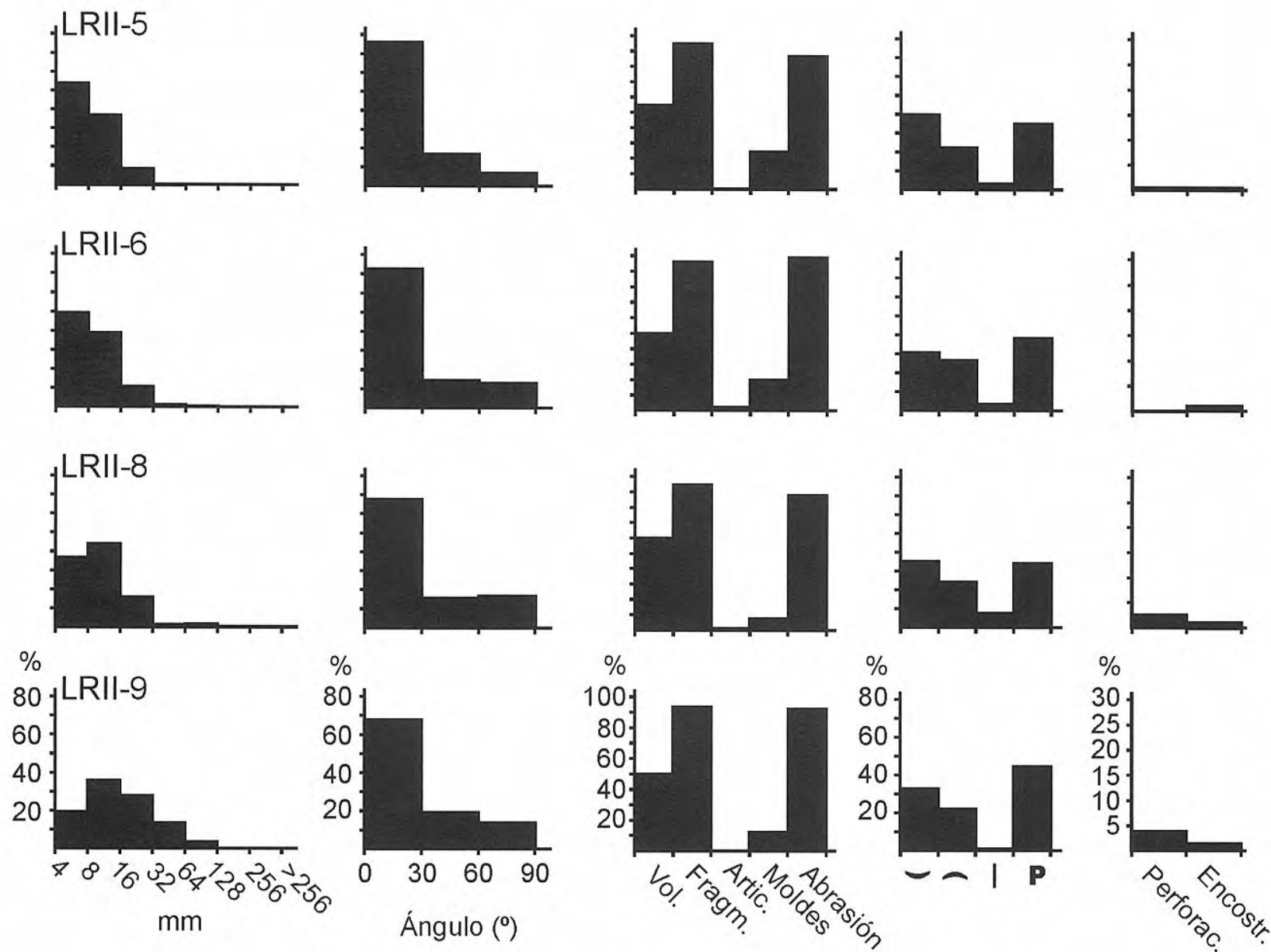

Figura 12. Histogramas de frecuencia de tamaños, inclinación de los bioclastos (biofábrica) y otras propiedades tafónomicas que caracterizan la agrupación B en la sección de Los Ranchos II. Abreviaturas como en la Fig. 4.

Histograms of the size-sorting, inclination of bioclasts (biofabric), and other taphonomic attributes characterising group B of the Los Ranchos II section. Abbreviations like in Fig. 4.

como la presencia de interacciones, son congruentes con un tiempo de exposición prolongado (Fürsich y Pandey, 1999; Behrensmeyer et al., 2000; Holland, 2000). Se trataría de un pavimento de Neopycnodonte formado en un medio de baja energía (probablemente plataforma externa) durante una parada relativa en la tasa de sedimentación.

Cabe señalar que en la base de este pavimento se encuentran acumulaciones de conchas en vaina con un ordenamiento muy parecido al descrito por SalazarJiménez et al. (1982) para los rellenos de galerías. Se trata de galerías subverticales abiertas por arriba en cuyas paredes se disponen las conchas subverticales y paralelas con la concavidad hacia arriba, mientras que en el fondo se acumulan las valvas, sobre todo, horizontalmente y con la concavidad hacia arriba (Fig. 8). En definitiva, dichas acumulaciones se interpretan como rellenos de madrigueras. Asimismo, atendiendo a las propiedades tafonómicas, se observa que muestran un patrón de concavidades e inclinaciones parecido al que SalazarJiménez et al. (1982) obtuvieron estudiando galerías aisladas rellenas de conchas. Es decir, hay un predominio de conchas orientadas con la concavidad hacia arriba, dispuestas en posición concordante y subvertical.
Puesto que estas madrigueras actúan como trampas de sedimento, esto explica el alto porcentaje de conchas completas que se observa, sobre todo en una de ellas (LRII-2-p2; Tabla 2), y la gran concentración de bioclastos por volumen de roca. Este es también el motivo de que estas acumulaciones muestren una distribución de tamaños muy dispersa, puesto que hay una menor fragmentación debido a que las conchas están menos expuestas a los agentes bioestratinómicos. Sin embargo, el alto porcentaje de redondeamiento junto con la presencia de perforaciones y organismos encostrantes, hace pensar que estos procesos debieron producirse cuando las conchas se encontraban en el exterior de la madriguera. Esto mismo es aplicable para la ausencia de articulación. Por todas estas razones, se podría inferir un relleno pasivo de las madrigueras en el que no actuaron los organismos que las construyeron (Martinell y Domènech, 1990). Este efecto trampa, donde se mezclan restos recientemente producidos y otros que han permanecido durante más tiempo expuestos sobre la interfase agua-sedimento, hace que no todas las estaciones de muestreos localizadas en los rellenos de madrigueras queden unidas en el análisis cluster (Yesares-García y Aguirre, 2002). 



Figura 13. Histogramas de frecuencia de tamaños, inclinación de los bioclastos (biofábrica) y otras propiedades tafónomicas que caracterizan la agrupación C en la sección de Los Ranchos II. Abreviaturas como en la Fig. 4.

Histograms of the size-sorting, inclination of bioclasts (biofabric), and other taphonomic attributes characterising group C of the Los Ranchos II section. Abbreviations like in Fig. 4.

E1 segundo tramo comienza con los niveles de carbonatos. La presencia de laminaciones cruzadas en artesa, junto con la alta fragmentación y total desarticulación, permiten inferir un aumento de energía en el medio. La gran abundancia de bioclastos que se encuentra y la alta concentración de algas sugieren que la tasa de sedimentación debió ser relativamente baja con escasez de sedimento en suspensión. Hay un claro predominio de las conchas en posición concordante, lo cual es congruente con la alta energía inferida anteriormente. La distribución de orientaciones de las valvas muestra una clara predominancia de conchas con las concavidades hacia arriba y, a su vez, un predominio de éstas sobre las verticales. En este tipo de ambientes, con estructuras de corrientes y alta energía, las conchas tenderían a encontrarse con la concavidad hacia abajo, puesto que es la posición hidrodinámicamente más estable. No obstante, el fuerte oleaje vuelca las conchas y las acumula, con la concavidad preferentemente hacia arriba, en los surcos de los ripples. Al caer en su interior sedimento, éste actúa a modo de lastre, impidiendo que adopten de nuevo la posición de estabilidad. Cuando los ripples migran se produce el enterramiento de las conchas acumuladas en los surcos, conservándose en su mayoría con la concavidad orientada hacia arriba (Clifton y Boggs, 1970; Clifton, 1971). El conjunto de propiedades tafonómicas, junto con los rasgos sedimentológicos y la litología, permiten inferir un ambiente de depósito somero de plataforma interna.

Dentro de las calcarenitas-calcirruditas se reconocen concentraciones residuales de fósiles en la base de cuerpos canaliformes. La distribución de tamaños de bioclastos dentro de estos canales muestra una importante dispersión, con el centro de la distribución localizado generalmente en clases de tamaños superiores a $20 \mathrm{~mm}$. Este patrón de distribución es debido a que la asociación fosilífera está formada, en su mayor parte, por conchas de gran tamaño (pectínidos y ostreidos). El sesgo que se observa hacia la izquierda de la distribución de tamaños es debido al proceso de lavado del material más fino de la matriz por la acción de corrientes. Pero dicho sesgo no afecta solamente a la distribución de tamaños, sino que también se ha detectado en el tipo de valvas que se encuentran mayoritariamente en estas acumulaciones. En el caso de los canales de la Calcarenita III de la sección de Los Ranchos I, del total de bioclastos sólo se ha podido identificar el tipo de valva en un porcentaje pequeño (10\%). De estos, el 70,1\% corresponden a las valvas cóncavas (valvas derechas en los pectínidos e izquierdas en los ostreidos). Esto se debe a que las valvas cóncavas, por su morfología y su peso, son las que primero se depositan al perder fuerza el flujo, mientras que las valvas planas continúan su camino con el resto de bioclastos de menor tamaño y junto al resto de la matriz. Esto indica, por tanto, que estas acumulaciones son el reflejo de un flujo de alta energía, probablemente relacionado con eventos de tormentas (Seilacher y Aigner, 1991). El alto porcentaje de abrasión en las conchas está directamente conectado con la alta energía inferida para el depósito de estos materiales. De igual forma, el predominio de conchas con la concavidad orientada hacia arriba es característica de depósitos formados a partir de flujos turbulentos de alta densidad, como por ejemplo los eventos de tormentas (Clifton, 1971; Speyer y Brett, 1988; Kidwell y Holland, 1991; Kidwell y Bosence, 1991). En el caso de los bivalvos, cuando el flujo pierde fuerza caen con la concavidad hacia arriba que es la posición de caída más estable (Allen, 1990; Seilacher y Aigner, 1991).

La diferenciación de estos cuerpos canalizados dentro de la matriz calcarenítica-calcirrudítica permite hacer la diferenciación de los procesos sedimentarios. Así, los canales representarían los depósitos eventuales mientras que la matriz reflejaría la sedimentación normal (events and background deposits respectivamente según Speyer y Brett, 1991). Durante las condiciones normales parece 
S



Figura 14. Esquema dibujado a partir de una fotografía de campo que muestra una panorámica de la zona de estudio. Obsérvese que en la mitad inferior del corte las capas se inclinan hacia el $\mathrm{N}$, mientras que en la superior, los paquetes de calcarenitas-calcirruditas lo hacen hacia el S. Este cambio en la geometría marca el inicio de la progradación de las facies de plataforma interna sobre los materiales depositados en ambientes de plataforma externa. El cambio de inclinación de las capas pudo estar relacionado con la actividad tectónica asociada a la zona de fallas de Carboneras, situada al SE de la zona de estudio.

Sketch from a field picture showing a panoramic view of the study area. Beds in the lower half of the section dip to the $N$, while calcarenitic-calciruditic beds of the upper half dip to the S. This change in the geometry of the sediments corresponds with the beginning of the progradation of the inner-shelf facies on sediments deposited in an outer platform. This change, in turn, could be associated with the tectonic activity of the Carboneras fault system, located SE of the study area.

que los procesos tafonómicos que prevalecen están asociados a un prolongado tiempo de exposición de las conchas sobre el fondo. Por el contrario, durante los eventos de tormentas los restos producidos estuvieron principalmente afectados por las condiciones de alta energía asociadas con las tormentas.

En la Calcarenita III las concentraciones fosilíferas en la base de canales aparecen frecuentemente amalgamadas, lo que denota una sedimentación en una zona de plataforma interna proximal (Seilacher y Aigner, 1991). Se interpreta como un depósito en ambiente submareal somero. Por tanto, la Calcarenita III se correspondería con el depósito más somero de la unidad pliocena en la zona de estudio. Además de por los rasgos sedimentológicos, su carácter somero también se manifiesta en la asociación fosilífera, dominada por Ostrea lamellosa y pectínidos de gran tamaño, como Flabellipecten bosniasckii o Gigantopecten. Como se ha comentado anteriormente, este banco carbonatado internamente presenta una estratificación cruzada que indica un sentido de paleocorriente hacia el N. Por todo ello, se interpreta como una barra calcarenítica que migraba hacia la paleocosta y que estuvo afectada por el efecto del oleaje de tormentas que produjo, localmente, la erosión de la barra y el depósito de conchas en la base de canales.

En la horizontal también se observa que la amalgamación de las acumulaciones fosilíferas en la base de canales es más frecuente en los paquetes carbonatados de Los Ranchos II. Así mismo, las Calcarenitas II y III, individualizadas en la sección de Los Ranchos I, se amalgaman hacia el $\mathrm{N}$, originándose un único banco carbonatado en la sección Los Ranchos II. Dicha amalgamación se produce por el acuñamiento hacia el $\mathrm{N}$ de las arenas intercaladas entre los dos paquetes (Fig. 14). Todo esto permite interpretar una somerización lateralmente hacia el $\mathrm{N}$.

Finalmente, las arenas intercaladas entre los niveles de calcarenitas-calcirruditas son cuerpos masivos, sin estructuras sedimentarias, en los que vuelven a ser dominantes los organismos de ambientes profundos como Neopycnodonte cochlear (Pérès, 1989; Poppe y Goto, 1993). Parecen corresponder, por tanto, a pulsos de profundización relativa entre etapas de condiciones más someras representadas por los cuerpos carbonatados. Cabe resaltar, no obstante, el tramo de arenas bioclásticas intercalado entre las Calcarenitas I y II de la sección Los Ranchos II. En estos materiales las conchas aparecen en concentraciones más o menos dispersas o bien delimitando galerías. Se interpreta como un depósito de concentración biogénica con una alta tasa de bioturbación en el que diferentes organismos excavadores realizaron madrigueras. La intensa actividad bioturbadora produjo la superposición y amalgamación de madrigueras, dando lugar a un sedimento con una bioturbación de fondo donde se distinguen rellenos discretos de madrigueras (Yesares-García y Aguirre, 2002).

En definitiva, de la discusión realizada se desprende la existencia de tres tipos de facies. Por un lado, las facies de limos y arenas masivas que representan depósitos de ambientes profundos y de baja energía formados, probablemente, en ambientes de plataforma externa por debajo del oleaje de tormentas. Dentro de estos depósitos se encuentran los rellenos bioclásticos en el interior de madrigueras. Finalmente, están los sedimentos carbonatados, interpretados como depósitos en medios de alta energía y por encima del oleaje de tormentas. Así mismo, los análisis cluster de las propiedades 


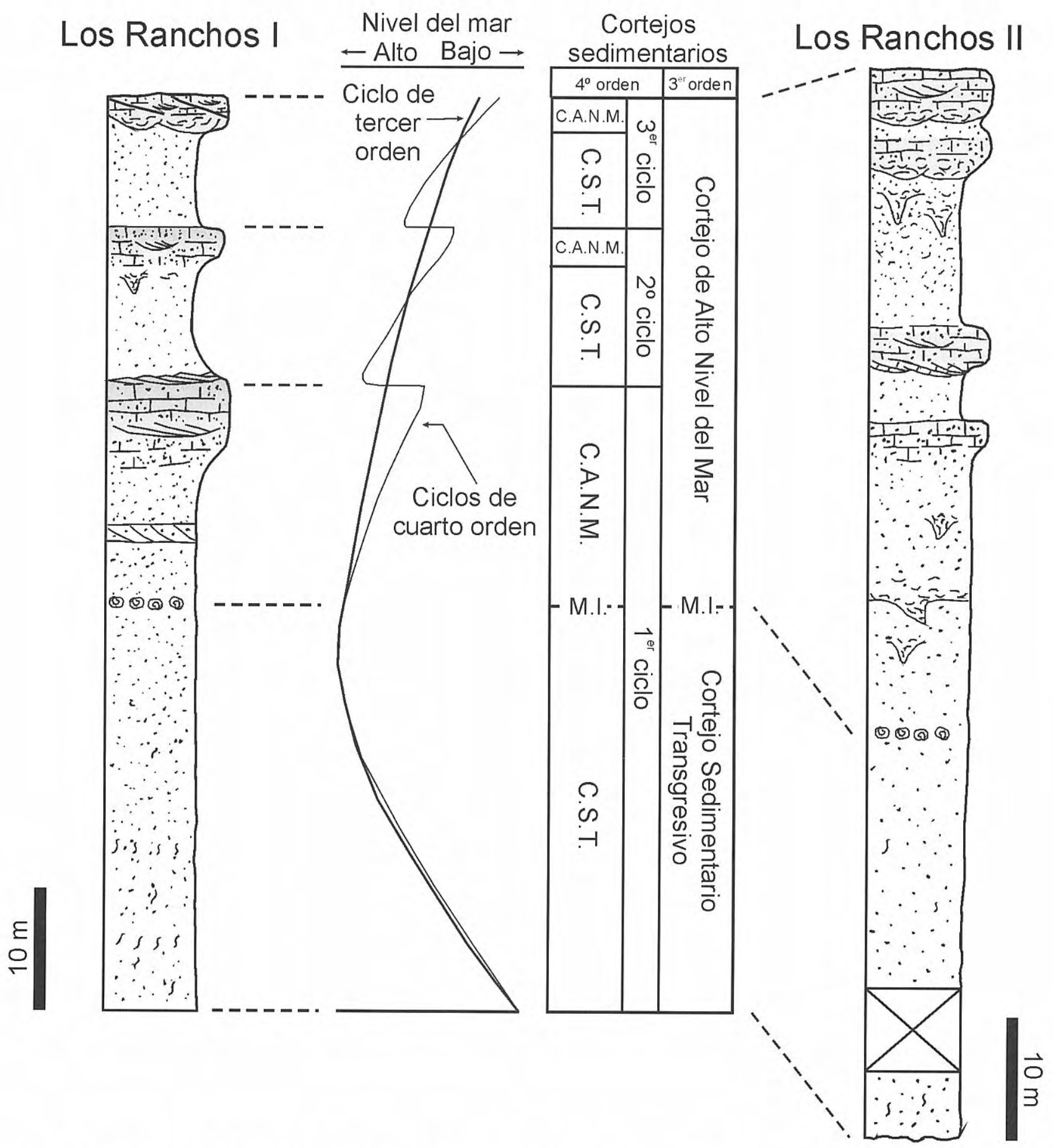

Figura 15. Variación del nivel del mar y ciclos sedimentarios deducidos a partir de la geometría de los estratos y de la interpretación paleoambiental inferida de las facies y las propiedades tafonómicas.

Sea-level changes and sedimentary cyclicity inferred from the geometry of the sediments and from the palaeonvironmental interpretation of the studied materials based on facies and taphonomic signatures.

tafonómicas (Figs. 3 y 9) ponen de manifiesto que las diferentes agrupaciones y subagrupaciones definidas reflejan estas mismas facies. El hecho de que se puedan definir grupos de muestras caracterizadas por las propiedades tafonómicas de los fósiles que contienen y que, además, tiene un significado paleoambiental concreto da validez, en última instancia, a los modelos de tafofacies (Yesares-García y Aguirre, 2002).

\section{ARQUITECTURA ESTRATIGRÁFICA Y MODELO SECUENCIAL}

Como se ha visto en la interpretación de las dos secciones estudiadas, la evolución vertical observada en el conjunto plioceno se caracteriza por una somerización hacia techo. Se pasa de facies de plataforma externa, situadas por debajo del nivel de acción del oleaje de 
tormentas, en la base, a facies de plataforma interna y ambientes submareales muy energéticos afectados por tormentas, en el techo. Una evolución somerizante similar a lo largo del Plioceno Inferior ha sido observada en otros puntos de la cuenca de Almería-Níjar (Montenat et al., 1990; Aguirre, 1995a, 1996, 1998; Aguirre et al., 1996), así como a lo largo de todo el litoral español (Montenat, 1977; Martinell, 1988; González-Delgado et al., 1992, 1993; Aguirre, 1995a, 1995b, 2000).

Los materiales estudiados se depositaron sobre una superficie irregular (Fig. 14), de modo que la paleotopografía debió controlar en gran medida la geometría de los materiales y la sedimentación en esta zona de la cuenca, dando lugar a rápidas transiciones de facies y fuertes cambios de espesor en los depósitos pliocenos. Esta paleotopografía también debió condicionar la posición del depocentro en el sector estudiado de modo que, durante la sedimentación del primer tramo de la Unidad I, dicho depocentro se encontraba al $\mathrm{N}$ de Los Ranchos II, en donde la cota de la paleotopografía era mínima. Esto también se observa por la geometría de las capas, inclinadas hacia el $\mathrm{N}$, y por la potencia del conjunto de materiales, que en esta zona es máxima y hacia el S (hacia Los Ranchos I y la Loma de Ciscar) disminuye. Por el contrario, a partir de la Calcarenita I se observa un cambio en la geometría de las capas ya que, desde aquí y hacia el techo, están inclinadas hacia el S (Fig. 14).

Las arenas-limos masivos del tramo inferior se corresponden con un medio de plataforma externa poco energético y con una tasa de sedimentación altamoderada. No obstante, localmente se detectan momentos de baja tasa de sedimentación o, incluso, de parada en la sedimentación. Este es el caso del nivel de rodolitos, en el que abundan los fragmentos de huesos de mamíferos marinos, que se extiende lateralmente entre las dos secciones.

En la Loma de Ciscar, al S de Los Ranchos I, aparece un tramo con estratificaciones cruzadas en artesa inmediatamente por encima del nivel de rodolitos. Hacia el $\mathrm{N}$ de Los Ranchos I, sin embargo, las estructuras sedimentarias desaparecen pasándose a arenas masivas donde se encuentra el pavimento de Neopycnodonte cochlear con la base modificada por la acción de organismos bioturbadores. Este cambio lateral de facies se interpreta como el paso hacia ambientes más profundos y de menor energía hacia el $\mathrm{N}$ que pudo estar controlado por la paleotopografía del fondo de la cuenca. De esta forma se infiere la existencia de un alto fondo relativo en la zona de la Loma de Ciscar (no emergida).

A partir del segundo tramo la geometría de los estratos cambia, de forma que se inclinan hacia el S (Fig. 14). La intercalación de los paquetes carbonatados refleja la instalación de las facies de plataforma interna, cada vez más someras según se sube en la secuencia. Así mismo, como se ha visto en la interpretación paleoambiental, durante el depósito del segundo tramo las facies más someras se localizaban hacia el extremo $\mathrm{N}$ del afloramiento (Los Ranchos II), mientras que las más profundas lo hacían hacia el S (Los Ranchos I). Este dispositivo proximal-distal, junto con la arquitectura geométrica observada, permite inferir que el segundo tramo corresponde con un sistema de plataforma carbonatada somera progradante hacia el $\mathrm{S}$ sobre facies más distales (Fig. 14). Unos metros por debajo de la Calcarenita I en Los Ranchos II se intercala un paquete calcarenítico (Figs. 2 y 14) que transiciona lateralmente hacia el $\mathrm{S}$ a arenas bioclásticas. Dicho paquete se interpreta como la primera señal sedimentaria del avance del conjunto progradante de las facies de plataforma interna representadas por los niveles de calcarenitas y calcirruditas del segundo tramo.

El dispositivo geométrico del conjunto plioceno, junto con la evolución paleoambiental deducida de las facies, las asociaciones de fósiles y de las propiedades tafonómicas, permite proponer un modelo de evolución secuencial para este periodo de tiempo en esta zona de la cuenca de Almería-Níjar. Según la evolución paleoambiental inferida, los limos y arenas del primer tramo se adaptan y van rellenando las zonas deprimidas de forma que llegan a rebasar los altos topográficos. Esto sugiere que este primer tramo pueda corresponderse con los sedimentos del cortejo sedimentario transgresivo (Fig. 15).

En la parte media de la unidad pliocena se encuentran el nivel de rodolitos (en ambas secciones estudiadas) y el pavimento de Neopycnodonte en la sección de Los Ranchos II. Como se ha comentado anteriormente, la formación de ambos niveles debió estar asociada con una ralentización, o incluso parada, en la tasa de sedimentación. Estas condiciones ambientales suelen estar asociadas con el evento de máxima inundación (Norris, 1986; Posamentier y Vail, 1988; Posamentier et al., 1988; Vail et al., 1991; Kidwell, 1991a, 1991b, 1993; Brett, 1995, 1998; Holland, 2000). Por tanto, es factible interpretar que la máxima inundación se alcanzara hacia la mitad del depósito de la unidad pliocena estudiada, bien coincidiendo con el depósito del nivel de rodolitos o con la formación del pavimento de Neopycnodonte (Fig. 15).

Finalmente, la geometría progradante de los sedimentos del segundo tramo, y su carácter cada vez más somero hacia techo, deducido de las facies, las estructuras sedimentarias y las asociaciones de fósiles, sugieren que este tramo representaría los depósitos del cortejo sedimentario de alto nivel de mar (Fig. 15).

El límite entre los dos tramos definidos, que representan dos etapas de la evolución secuencial inferida, es erosivo y trunca a los materiales infrayacentes (Fig. 14). Esto es especialmente evidente en la sección de Los Ranchos II, donde la Calcarenita I muestra una base erosiva con concentraciones de fósiles en la base de los canales. Este truncamiento y cambio en la evolución secuencial de la Unidad I del Plioceno en la zona estudiada puede estar relacionado con un proceso tectónico asociado al sistema de fallas del accidente de Carboneras o a un pulso de levantamiento de Sierra Cabrera, el margen $\mathrm{N}$ de la cuenca durante el depósito de esta unidad (Aguirre 1998; Braga et al., 2001, 2003). Ambos procesos pueden ser complementarios, no tienen por qué ser excluyentes. 
Los paquetes arenosos masivos del segundo tramo representan pulsos de profundización con respecto a los paquetes carbonatados. Esta alternancia puede interpretarse como una ciclicidad interna de mayor frecuencia, probablemente de cuarto orden. Así, cada ciclo comienza con arenas y finaliza con los paquetes calcareníticos (Fig. 15). En el caso de las arenas bioclásticas intercaladas entre los dos primeros bancos carbonatados en Los Ranchos II se observa una geometría retrogradante (Fig. 14). Esto permite inferir que las arenas corresponderían a las facies del cortejo sedimentario transgresivo mientras que las calcarenitascalcirruditas reflejarían los depósitos del cortejo sedimentario de alto nivel de mar.

\section{CONCLUSIONES}

La Unidad I del Plioceno estudiada en el sector de El Argamasón (NE de la cuenca de Almería-Níjar) está representada por una secuencia somerizante a techo. Se pasa de limos y arenas depositados en ambientes de plataforma externa a calcarenitas y calcirruditas formadas en ambientes de plataforma interna. Este cambio paleoambiental se evidencia, además de por un cambio litológico y en las facies, por cambios en las asociaciones de organismos y en las propiedades tafonómicas de los fósiles. Así, los depósitos profundos están dominados por Korobkovia, Amusium, y Neopycnodonte, mientras que las facies de plataforma interna están caracterizadas por Ostrea lamellosa y pectínidos de gran tamaño, con fuertes costillas y una fuerte curvatura de la valva (Gigantopecten, Flabellipecten bosniasckii).

Desde el punto de vista tafonómico, los sedimentos de plataforma externa están caracterizados por: a) una baja concentración de bioclastos; b) empaquetamiento denso a poco denso; c) interacciones biológicas frecuentes; d) corrasión media a alta; e) distribución de ángulos bimodal con predominio de las posiciones concordante y perpendicular; y f) una tendencia a la aleatoriedad en la orientación de las concavidades. Por otro lado, en los ambientes de plataforma interna se registra un empaquetamiento denso, con una alta concentración de bioclastos y una alta abrasión. La selección de tamaños es pobre y la distribución de ángulos es unimodal con claro predominio de las conchas en posición concordante. Finalmente, las concentraciones fosilíferas en el interior de madrigueras, o los sedimentos intensamente bioturbados se caracterizan por presentar un empaquetamiento denso, una alta abundancia, una escasa selección de tamaños y una distribución bimodal de ángulos con cierta tendencia a la aleatoriedad. Predominan las conchas con la concavidad hacia arriba.

El dispositivo geométrico, junto con los cambios en las facies y en las asociaciones de fósiles, indican que la primera mitad de la unidad estudiada se depositó rellenando un paleorrelieve excavado en el substrato mioceno con un alto fondo relativo (no emergido) en el extremo $\mathrm{S}$ del afloramiento y una zona más profunda hacia el N. Por el contrario, en la parte superior se estableció un sistema de plataforma carbonatada progradante hacia el S. Este sistema de plataforma carbonatada progradante erosiona y trunca a los sedimentos infrayacentes. Por tanto, dicho cambio se asocia con una posible reactivación del basamento bético que constituyó el margen $\mathrm{N}$ de la cuenca (Sierra Cabrera) o por un pulso tectónico en el sistema de fallas del accidente de Carboneras, localizado inmediatamente al ESE de la zona de estudio. Ambos procesos no son excluyentes, sino que pueden ser complementarios.

Desde un punto de vista secuencial, la Unidad I del Plioceno se caracteriza por un ciclo sedimentario en el que se infiere una profundización hasta la parte media de la unidad seguida de una somerización hacia techo. Esto ha permitido reconocer un cortejo sedimentario transgresivo y un cortejo sedimentario de alto nivel separados por un nivel de algas coralináceas que se ha interpretado como el depósito formado durante la máxima inundación. Internamente, la secuencia pliocena puede dividirse en tres ciclos sedimentarios de mayor frecuencia que muestran también una tendencia de profundización seguida de una somerización.

\section{AGRADECIMIENTOS}

Queremos expresar nuestro agradecimiento a la Dra. Isabel $M^{a}$ Sánchez-Almazo por sus sugerencias y su colaboración en las fases iniciales del desarrollo de este trabajo. Así mismo, agradecemos las revisiones realizadas por los Drs. Jordi Martinell y Sixto Fernández López, así como las sugerencias editoriales de la Dra. María Luisa Martínez. Este trabajo ha estado financiado por los Proyectos de Investigación PB97-0809 y BTE2001-3023 del Ministerio de Ciencia y Tecnología, y por el Grupo de Investigación de la Junta de Andalucía denominado "Paleogeografía de Cuencas Sedimentarias".

\section{BIBLIOGRAFÍA}

Aguirre, J., 1995a. Tafonomía y Evolución Sedimentaria del Plioceno Marino en el Litoral sur de España entre Cádiz y Almería. Tesis Doctoral, Universidad de Granada, 419 pp.

Aguirre, J., 1995b. Implicaciones paleoambientales y paleogeográficas de dos discontinuidades estratigráficas en los depósitos pliocénicos de Cádiz (SW de España).

- Revista de la Sociedad Geológica de España, 8, 161174.

Aguirre, J., 1996. Modelo de tafofacies en el Plioceno inferior de la Cuenca de Almería-Níjar (SE de España). In: II Reunión de Tafonomía y Fosilización (Eds. G. Meléndez Hevia, M.F. Blasco Sancho y I. Pérez Urresti), Zaragoza, 11-18.

Aguirre, J., 1998. El Plioceno del SE de la Península Ibérica (provincia de Almería). Síntesis estratigráfica, sedimentaria, bioestratigrafica y paleogeográfica. Revista de la Sociedad Geológica de España, 11, 297-315. 
Aguirre, J., 2000. Evolución paleoambiental y análisis secuencial de los depósitos Pliocenos de Almayate (Málaga, S de España). Revista de la Sociedad Geológica de España, 13, 45-57.

Aguirre, J., Braga, J.C., Jiménez, A.P. and Rivas, P. 1996. Substrate-related changes in pectinid fossil assemblages. Palaeogeography, Palaeoclimatology, Palaeoecology 126, 291-308.

Allen, J.R.L. 1990. Transport - Hydrodynamics. In: Palaeobiology: a Synthesis (Eds. D.E.K. Briggs y P.R. Crowther). Blackwell, Oxford, 227-230.

Behrensmeyer, A.K. and Kidwell, S.M. 1985. Taphonomy's contributions to paleobiology. Paleobiology, 11, 105119.

Behrensmeyer, A.K., Kidwell, S.M. and Gastaldo, R.A. 2000. Taphonomy and paleobiology. In: Paleobiology's Perspective (Eds. D.H. Erwing y S.L. Wing), The Paleontological Society, Lawrence, 26, 103-147.

Best, M.M.R. and Kidwell, S.M. 2000. Bivalve taphonomy in tropical mixed siliciclastic-carbonate settings. I. Environmental variation in shell condition. Paleobiology, 26, 80-102.

Boorsma, L.J. 1992. Syn-tectonic sedimentation in a Neogene strike-slip basin containing a stacked Gilberttype delta (SE Spain). Sedimentary Geology, 81, 105123.

Bosence, D.W.J. 1983. The occurrence and ecology of recent rhodoliths - A review. In: Coated Grains (Ed. T.M. Peryt), Springer-Verlag, Berlín, 225-242.

Braga, J.C., Martín, J.M. y Aguirre, J. 2001. Paleogeografía del SE de España en el Mioceno superior y Plioceno. XVII Jornadas de la Sociedad Española de Paleontología, 63-67.

Braga, J.C., Martín, J.M. and Quesada, C. 2003. Patterns and average rates of late Neogene-Recent uplift of the Betic Cordillera, SE Spain. Geomorphology, 50, 3-26.

Brett, C.E. 1995. Sequence stratigraphy, biostratigraphy and taphonomy in shallow marine environments. Palaios, 10, 597-616.

Brett, C.E. 1998. Sequence stratigraphy, paleoecology, and evolution: biotic clues and responses to sea-level fall. Palaios, 13, 241-262.

Brett, C.E. and Baird, G.C. 1986. Comparative taphonomy: a key to paleoenvironmental interpretation based on fossil preservation. Palaios, 1, 207-227.

Cadée, G.C. 1992. Eolian transport and left/right sorting of Mya shells (Mollusca, Bivalvia). Palaios, 7, 198-202.

Cadée, G.C. 1994a. Eider, shelduck, and other predators, the main producers of shell fragments in the Wadden sea: palaeoecological implications. Palaeontology, 37, 181202.

Cadée, G.C. 1994b. Mya shell manipulating by turnstones (Aves) results in concave-up position and left/right sorting. Palaios, 9, 307-309.

Callender, W.R., Staff, G.M., Powell, E.N. and Macdonald, I.R. 1990. Gulf of Mexico hydrocarbon seep communities V. Biofacies and shell orientation of autochtonous shell beds below storm wave base. Palaios, 5, 2-14.
Carter, R.M., 1972. Adaptations of British chalk bivalvia. Journal of Paleontology, 46, 325-341.

Clifton, H.E. 1971. Orientation of empty pelecypod shells and shell fragments in quiet water. Journal of Sedimentary Petrology, 41, 671-682.

Clifton, H.E. and Boggs, S., Jr. 1970. Concave-up pelecypod (Psephidia) shells in shallow marine sand, Elk River Beds, southwestern Oregon. Journal of Sedimentary Petrology, 40, 888-897.

Droser, M. and Bottjer, D.J. 1986. A semiquantitative field classification of ichnofabric. Journal of Sedimentary Petrology, 56, 558-569.

Droser, M. and Bottjer, D.J. 1989. Ichnofabric of sandstones deposited in high-energy nearshore environments: Measurement and utilization. Palaios, 4, 598-604.

Emery, K.O. 1968. Positions of empty pelecypod valves on the continental shelf. Journal of Sedimentary Petrology, 38, 1264-1269.

Fürsich, F.T. and Pandey, D.K. 1999. Genesis and environmental significance of Upper Cretaceous shell concentrations from the Cauvery Basin, southern India. Palaeogeography, Palaeoclimatology, Palaeoecology, 145, 119-139.

González-Delgado, J.A., Andrés, I. and Civis, J. 1992. Late Miocene and early Pliocene molluscan faunas from the northeast Atlantic (Lisboa, Algarve, W. Guadalquivir, NW Morocco). First Congress R.C.A.N.S., Lisboa, 5962.

González-Delgado, J.A., Sierro, F.J. y Civis, J. 1993. Cambios globales del nivel del mar y concentraciones de megafaunas marinas someras en el Neógeno del oeste de la Península Ibérica, IX Jornadas de Paleontología, Málaga, 33-36.

Gould, S.J. 1971. Muscular mechanics and the ontogeny of swimming in scallops. Palaeontology, 14, 61-94.

Goy, J.L. y Zazo, C. 1982. Niveles marinos cuaternarios y su relación con la tectónica en el litoral de Almería (España). Boletín de la Real Sociedad de Historia Natural (Geología), 80, 171-184.

Goy, J.L. and Zazo, C. 1986. Synthesis of the Quaternary in the Almeria littoral neotectonic activity and its morphologic features, western Betic, Spain. Tectonophysics, 130, 259-270.

Hickman, C.S. 1984. Composition, structure, ecology and evolution of six cenozoic deep-water mollusk communities. Journal of Paleontology, 58, 1215-1234.

Holland, S.M. 1995. The stratigraphic distribution of fossils. Paleobiology, 21, 92-109.

Holland, S.M. 2000. The quality of the fossil record: a sequence stratigraphic perspective. In: Paleobiology's Perspective (Eds. D.H. Erwing y S.L. Wing), The Paleontological Society, Lawrence, 26, 149-168.

Kidwell, S.M. 1985. Palaeobiological and sedimentological implications of fossil concentrations. Nature, 318, $457-$ 460.

Kidwell, S.M. 1986. Models for fossil concentrations: paleobiologic implications. Paleobiology, 12, 6-24.

Kidwell, S.M. 1988. Taphonomic comparison of passive and active continental margins: Neogene shell beds of the Atlantic coastal plain and northern Gulf of California. 
Palaeogeography, Palaeoclimatology, Palaeoecology, 63, 201-223.

Kidwell, S.M. 1989. Stratigraphic condensation of marine transgressive records: origin of major shell deposits in the Miocene of Maryland. Journal of Geology, 97, 1-24.

Kidwell, S.M. 1991a. Condensed deposits in siliciclastic sequences: expected and observed features. In: Cycles and Events in Stratigraphy (Eds. G. Einsele, W. Ricken and A. Seilacher). Springer-Verlag, Berlin, 682-695.

Kidwell, S.M. 1991b. The stratigraphy of shell concentrations. In: Taphonomy. Releasing the Data Locked in the Fossil Record (Eds. P.A. Allison and D.E.G. Briggs). Topics in Geobiology. Plenum Press, New York, 221-290.

Kidwell, S.M. 1993. Taphonomic expressions of sedimentary hiatus: field observations on bioclastic concentrations and sequence anatomy in low, moderate and high subsidence settings. Geologische Rundschau, 82, 189-202.

Kidwell, S.M. and Bosence, D.W.J. 1991. Taphonomy and time-averaging of marine shelly faunas. In: Taphonomy. Releasing the Data Locked in the Fossil Record (Eds. P.A. Allison and D.E.G. Briggs). Topics in Geobiology. Plenum Press, New York, 115-209.

Kidwell, S.M. and Holland, S.M. 1991. Field description of coarse bioclastic fabrics. Palaios, 6, 426-434.

Martinell, J. 1988. An overview of the marine Pliocene of N.E. Spain. Géologie Méditerranéenne, 15, 227-233.

Martinell, J. y Domènech, R. 1990. Las conchas de bivalvos como trampas pasivas en el registro fósil. In: I Reunión de Tafonomía y Fosilización (Coord. S. FernándezLópez), Madrid, 179-194.

Milliman, J.D. 1977. Role of calcareous algae in Atlantic continental margin sedimentation. In: Fossil Algae, Recent Results and Develpments (Ed. E. Flügel). Springer-Verlag, Berlín, 232-247.

Montenat, C. 1977. Les bassins Néogènes du Levante d'Alicante et de Murcia (Cordillères Bétiques orientales Espagne). Stratigraphie, paleogeographie et évolution dynamique. Documents de Laboratoire de Géologie, Facultad de Sciences de Lyon, 69, 345 pp.

Montenat, C., Ott d'Estevou, P. et Chappelle, G.d.L. 1990. Le bassin de Nijar-Carboneras et le couloir du BasAndarax. In: Les bassins Néogènes du domaine Bétique Oriental (Espagne) (Ed. C. Montenat). Documents et Travaux Institut Géologique Albert-de-Lapparent, Paris, 12-13, 129-164.

Norris, R.D. 1986. Taphonomic gradients in shelf fossil assemblages: Pliocene Purisima Formation, California. Palaios, 1, 256-270.

Parsons, K.M. and Brett, C.E. 1991. Taphonomic processes and biases in modern marine environments: an actualistic perspective on fossil assemblage preservation. In: The Processes of Fossilization (Ed. S.K. Donovan). Belhaven Press, London, 22-65.

Pérès, J.M. 1989. Historia de la biota mediterránea y la colonización de las profundidades. In: El Mediterráneo Occidental (Ed. R. Margalef). Omega, Barcelona, 200234.
Poppe, G.T. and Goto, Y. 1993. European Seashells, 2. Verlag Christa Hemmen, Wiesbaden, $221 \mathrm{pp}$.

Posamentier, H.W. and Vail, P.R. 1988. Eustatic controls on clastic deposition. II.- Sequence and systems tract models. In: Sea Level Changes - An Integrated Approach (Eds. C.K. Wilgus, B.S. Hastings, C.G.S.C. Kendall, H. Posamentier, C.A. Ross and J.C. Van Wagoner). SEPM Special Publication, 42, 125-154.

Posamentier, H.W., Jervey, M.T. and Vail, P.R. 1988. Eustatic controls on clastic deposition. I - Conceptual framework. In: Sea Level Changes - An Integrated Approach (Eds. C.K. Wilgus, B.S. Hastings, C.G.S.C. Kendall, H. Posamentier, C.A. Ross and J.C. Van Wagoner). SEPM Special Publication, 42, 109-124.

Powell, E.N., Staff, G.M., Davies, D.J. and Callender, W.R. 1989. Macrobenthic death assemblages in modern marine environments: formation, interpretation, and application. Aquatic Sciences, 1, 555-589.

Prager, E.J. 1987. The Growth and Structure of Calcareous Nodules (For-Algaliths) on Florida's Outer Shelf. University of Miami, $65 \mathrm{pp}$.

Salazar-Jiménez, A., Frey, R.W. and Howard, J. 1982. Concavity orientations of bivalve shells in estuarine and nearshore shelfs sediments, Georgia. Journal of Sedimentary Petrology, 52, 565-586.

Seilacher, A. and Aigner, T. 1991. Storm deposition at the bed, facies, and basin scale: the geologic perspective. In: Cycles and Events in Stratigraphy (Eds. G. Einsele, W. Ricken and A. Seilacher). Springer-Verlag, Berlin, 249267.

Serrano, F. 1979. Los Foraminíferos Planctónicos de la Cuenca de Ronda y su Comparación con los de Otras Áreas de las Cordilleras Béticas. Tesis Doctoral, Universidad de Málaga, 272 pp.

Speyer, S.E. and Brett, C.E. 1986. Trilobite taphonomy and middle Devonian taphofacies. Palaios, 1, 312-327.

Speyer, S.E. and Brett, C.E. 1988. Taphofacies models for epeiric sea environments: Middle Paleozoic examples. Palaeogeography, Palaeoclimatology, Palaeoecology, 63, 225-262.

Speyer, S.E. and Brett, C.E. 1991. Taphofacies controls: background and episodic processes in fossil assemblage preservation. In: Taphonomy. Releasing the Data Locked in the Fossil Record (Eds. P.A. Allison and D.E.G. Briggs). Topics in Geobiology. Plenum Press, New York, 501-545.

Stanley, S.M. 1972. Functional morphology and evolution of byssally attached bivalve mollusks. Journal of Paleontology, 46, 165-212.

Stanley, S.M. 1988. Adaptative morphlogy of the shell in bivalves and gastropods. In: The Mollusca. Vol. 11, Form and Function (Eds. E.R. Trueman and M.R. Clarke), Academic Press, 105-140.

Studencki, W. 1979. Sedimentation of algal limestones from Busko-Spa environs (middle Miocene, Central Poland). Palaeogeography, Palaeoclimatology, Palaeoecology, 27, 155-165.

Ten Hove, H.A. and Den Hurk, P.V. 1993. A review of recent and fossil serpulid 'reefs'; actuopaleontology and 
the 'upper Malm' serpulid limestones in NW Germany. Geologie in Mijnbouw, 72, 23-67.

Ten Hove, H.A. and Smith, R.S. 1990. A re-description of Ditrupa gracillima Grube, 1878 (Polychaeta, Serpulidae) from the Indo-Pacific, with a discussion of the genus. Records Australian Museum, 42, 101-118.

Vail, P.R., Audemard, F., Bowman, S.A., Eisner, P.N. and Pérez-Cruz, C. 1991. The stratigraphic signatures of tectonics, eustasy and sedimentology - An overview. In: Cycles and Events in Stratigraphy (Eds. G. Einsele, W.
Ricken and A. Seilacher), Springer-Verlag, Berlin, 617659.

Wilson, M.V.H. 1988. Taphonomic processes: information loss and information gain. Paleoscene, 15, 131-147.

Yesares-García, J. and Aguirre, J. 2002. A methodological procedure for taphonomic analysis and taphofacies models. An experimental approach and a case study. In: Current Topics on Taphonomy and Fossilization (Eds. M. De Renzi, M.V. Pardo Alonso, M. Belinchón, E. Peñalver, P. Montoya and A. Márquez-Aliaga). Valencia, 435-443.

Manuscrito recibido: 26 de junio, 2002 Manuscrito aceptado: 23 de octubre, 2002 